\title{
Wavelength Selective Generation of Aryl Radicals and Aryl Cations for Metal-free Photoarylations
}

\author{
Stefano Crespi, Stefano Protti, * Maurizio Fagnoni* \\ PhotoGreen Lab, Department of Chemistry, Viale Taramelli 12, 27100 Pavia, Italy \\ SUPPORTING INFORMATION
}

\section{CONTENT}

1. Experimental Section.

1.1 Photophysical data of arylazo sulfones $\mathbf{1 a - h} \quad$ S2

1.2 Optimization of the reaction conditions for the synthesis of $\mathbf{2 a}$ S7

2. Copy of ${ }^{1} \mathrm{H}$ and ${ }^{13} \mathrm{C}$ NMR of compounds $\mathbf{1 a - h ,} \mathbf{2} \mathbf{a}-\mathbf{h}, \mathbf{4 - 8}$.

S9 


\section{Experimental Section.}

\subsection{Photophysical data of arylazo sulfones 1a-h}

Table S1. Photophysical data of compounds 1a-h. ${ }^{a}$

\begin{tabular}{cc}
\hline Compound & $\begin{array}{c}\lambda_{\text {abs, }}, \mathbf{n m} \\
\left(\boldsymbol{\varepsilon}, \mathbf{M}^{\mathbf{1}} \mathbf{c m}^{-\mathbf{1}}\right)\end{array}$ \\
\hline 1a & $435(155) ;$ \\
& $288.5(15360)$ \\
1b & $424(215)$ \\
& $294(16870)$ \\
1c & $425(170)$ \\
& $305(17000)$ \\
1d & $427(135)$ \\
& $294(14530)$ \\
1e & $421.5(255)$ \\
& $310.5(14190)$ \\
1g & $425(230)$ \\
& $342(17405)$ \\
1h & $439(160)$ \\
& $289(17230)$ \\
& $437(95)$ \\
\end{tabular}

${ }^{a}$ The $\varepsilon$ value related to the low intensity band in the visible was determined by using $5 \times 10^{-4} \mathrm{M}$ solutions of $\mathbf{1 a - h}$ in neat acetonitrile, whereas $5 \times 10^{-5} \mathrm{M}$ solutions were used to determine the $\varepsilon$ value in the UV region. 
Figure S1. UV absorption spectra of compounds $1 \mathbf{a}-\mathrm{h}$ in $\mathrm{MeCN}\left(5 \times 10^{-5} \mathrm{M}\right)$
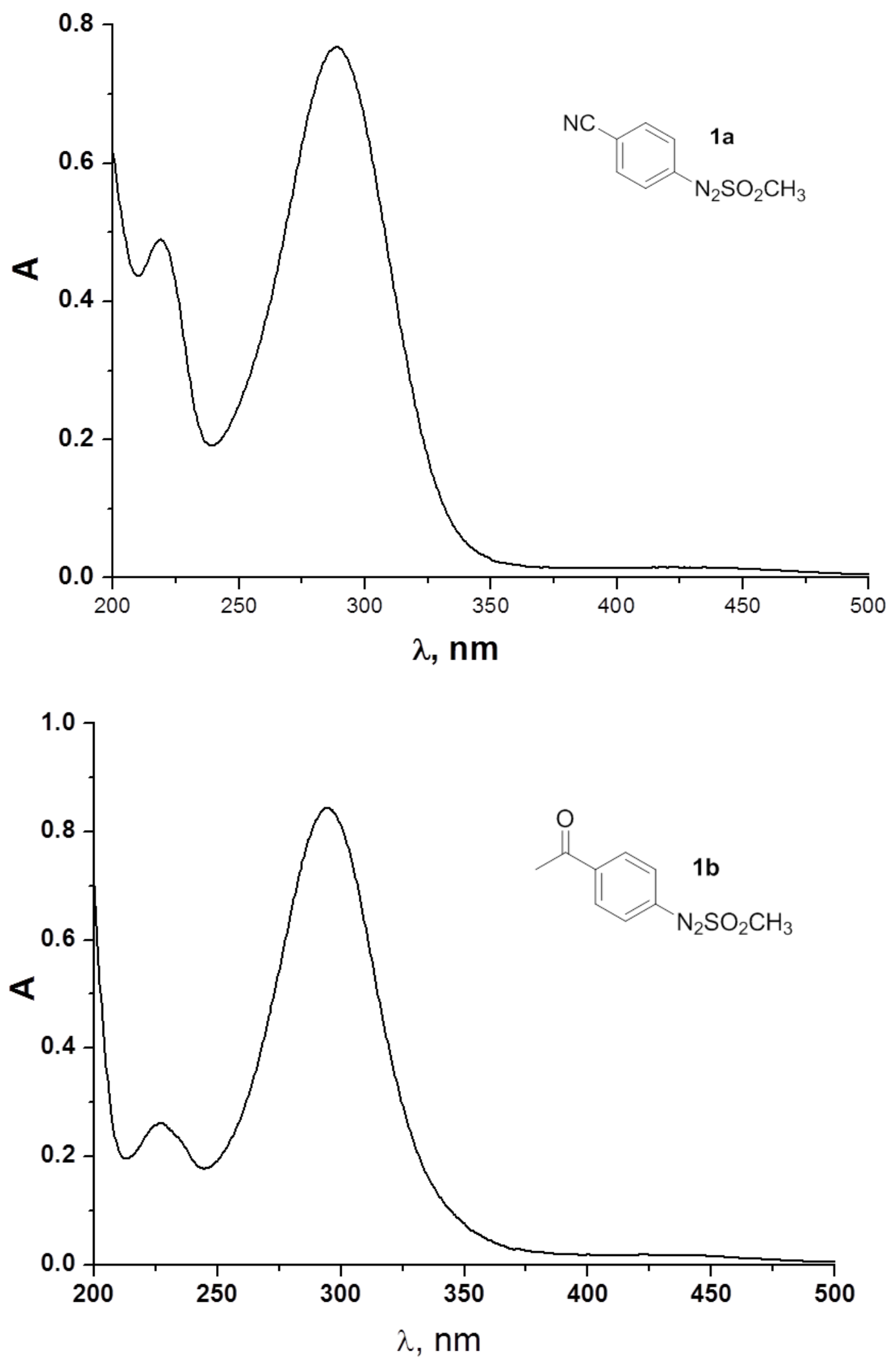

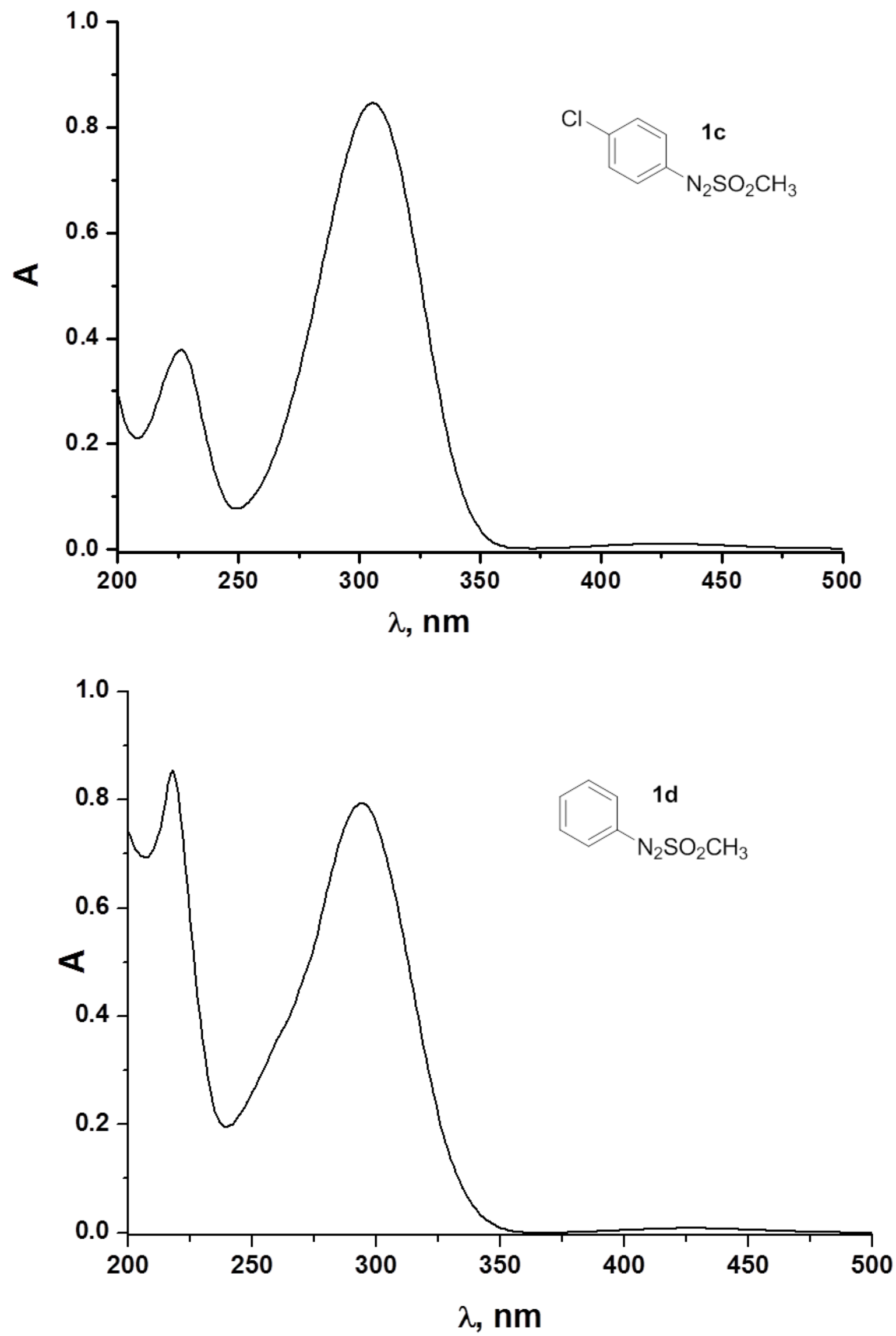

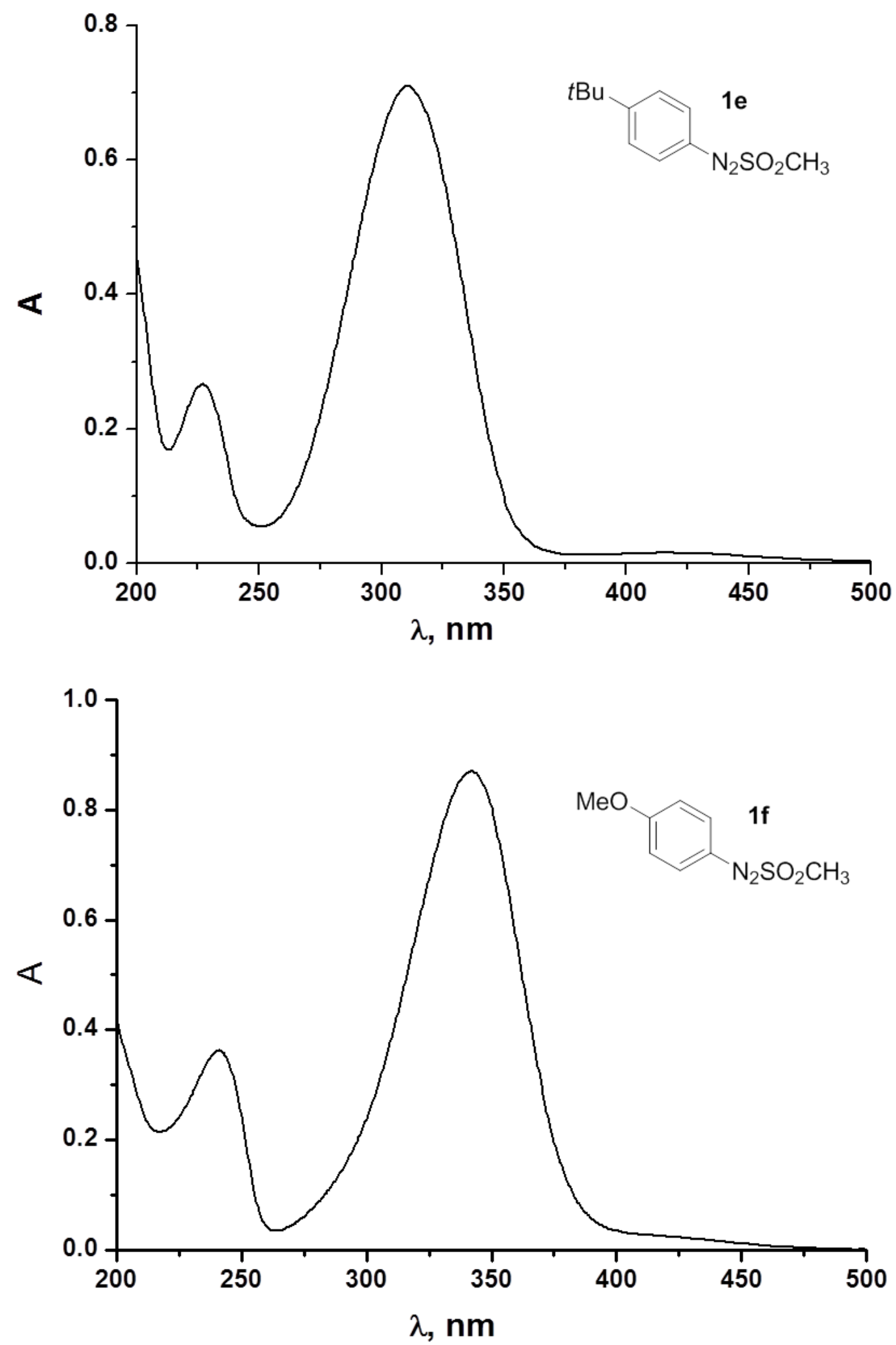

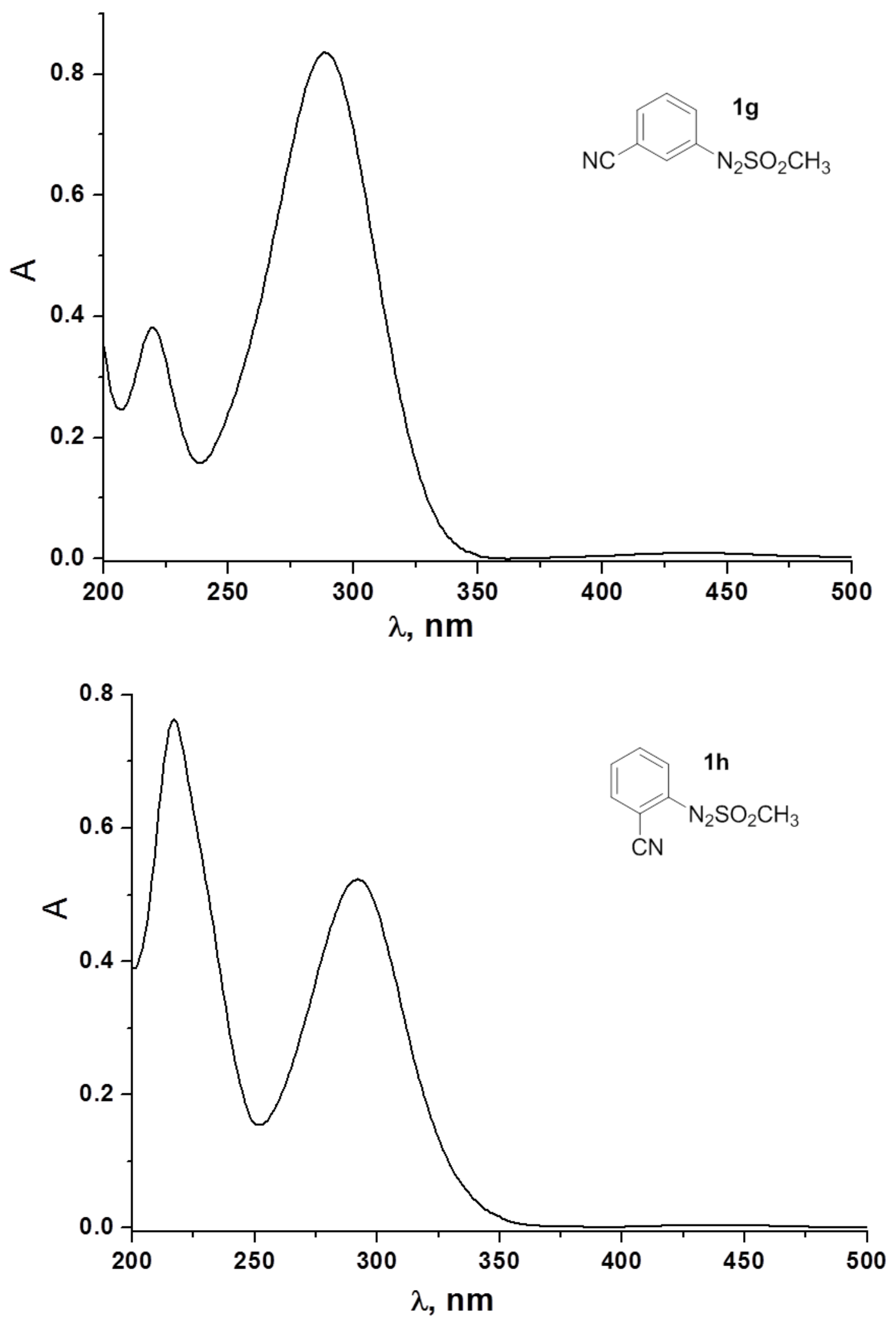


\subsection{Optimization of the reaction conditions for the synthesis of $2 \mathrm{a}$.}

Preliminary experiments were carried out by using arylazosulfone $\mathbf{1 a}$ as the model compound. Thus, degassed solutions of the substrate $(0.05 \mathrm{M})$ were irradiated in the presence of furan $(2 \mathrm{M})$. Since the UV-Vis absorption spectra of arylazo sulfones significantly overlaps the short wavelength of the sunlight spectrum at the earth's surface, reactions were performed by using nitrogen-purged solutions in a Pyrex glass vessel $(5 \mathrm{~mL})$ sealed with a rubber septum reported in Figure $\mathrm{S} 2$ using a solar light simulator apparatus (Solarbox 1500e COFOMEGRA Xe lamp, conditions: $500 \mathrm{~W} / \mathrm{m}^{2}$ of irradiance, filter: IR + outdoor) as the light source. In some instances irradiations were carried out by means of a phosphor coated Hg lamp $(15 \mathrm{~W}, \lambda=366 \mathrm{~nm})$ or a $\operatorname{LED}(1 \mathrm{~W}, \lambda=450 \mathrm{~nm})$.

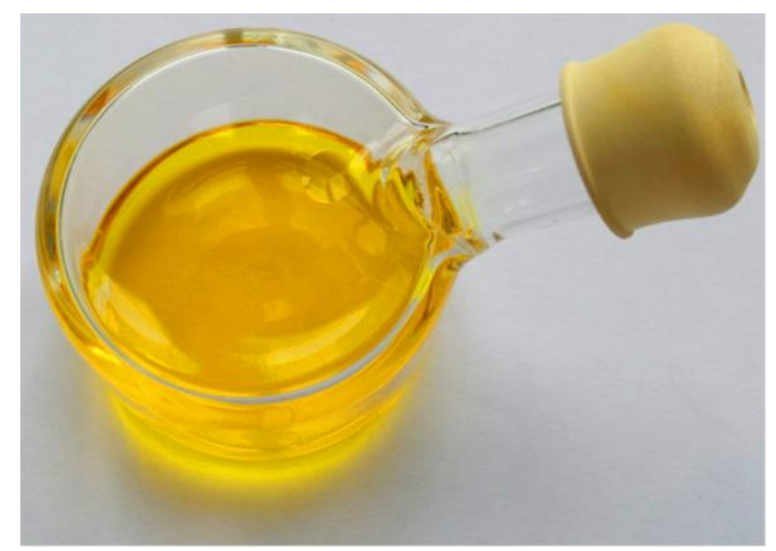

Figure S2. Pyrex vessel containing a $0.05 \mathrm{M}$ solution of $\mathbf{1 a}$.

The obtained results are summarized in Table S2. Two competing processes have been observed, namely arylation of furan to afford heterobiaryl $\mathbf{2 a}$ and reductive elimination of the azosulfone moiety to give benzonitrile 3a. Reduction to 3a was predominant in media such as dichloromethane and 1,4-dioxane (entries 1,2). The use of a 1,4-dioxane-water 9:1 mixture (entry 3) led to a slight improvement of the $\mathbf{2 a / 3 a}$ ratio. Higher amount of water (entry 4), however, made the arylation less clean. 2a was obtained in good yields in ethyl acetate, diethyl carbonate and acetone (entries 5-7), along with a significant amount of 3a. The use of an acetone--water 9:1 mixture did not show any substantial changes in the process. Protic media such as methanol, methanol/water mixture and 2,2,2-trifluoroethanol (TFE) (entries 9:12) led to unsatisfying results (in TFE, GC analyses also pointed out the formation of 4-fluorobenzonitrile in $12 \%$ yield). Irradiation of 1a in acetonitrile afforded discrete amount of $\mathbf{2 a}$ (46\% yield, entry 13) along with 5\% of 3a. However, when moving to acetonitrile/water 9:1 mixture (entry 14), arylation to $\mathbf{2 a}$ took place selectively (61\% yield). A similar yield of $\mathbf{2 a}(56 \%$, entry 15$)$ was likewise observed when exposing the solution to natural 
sunlight ( 3 days, 8 hours a day insulation). As observed in the case of acetone, higher amounts of water (entry 16) led to a strong decrease in the 2a yield. These results encouraged us to consider an acetonitrile/water 9:1 mixture as the preferred reaction medium in the following experiments. Gratifyingly, when doubling the concentration of $\mathbf{1 a}(0.1 \mathrm{M}), 2$-arylfuran $\mathbf{2 a}$ was obtained in $78 \%$ yield (entry 17), along with a small amount of 4-cyanophenyl acetanilide 9a (6\% yield). A decrease in the concentration of furan (1 M) still gave $\mathbf{2 a}$ in a satisfying amount (70\% yield, entry 18).

Table S2. Irradiation of $1 \mathrm{a}$ in the presence of furan.

\begin{tabular}{|c|c|c|c|c|}
\hline $1 \mathrm{a}$ & $\mathrm{M}_{\mathrm{N}^{\prime \prime}} \mathrm{O}_{\mathrm{O}^{\prime \prime} \mathrm{S}^{\prime \prime}}^{\mathrm{O}} \mathrm{CH}_{3}$ & & CN & $\mathrm{NHCOCH}_{3}$ \\
\hline Entry & Solvent & $\begin{array}{c}2 a \\
(\% \text { yield })\end{array}$ & $\begin{array}{c}3 a \\
\text { (\% yield })\end{array}$ & $\begin{array}{c}9 \mathbf{a} \\
(\% \text { Yield })\end{array}$ \\
\hline 1 & $\mathrm{CH}_{2} \mathrm{Cl}_{2}$ & 11 & 54 & - \\
\hline 2 & 1,4-dioxane & 38 & 43 & - \\
\hline 3 & 1,4-dioxane- $\mathrm{H}_{2} \mathrm{O} 9: 1$ & 40 & 10 & - \\
\hline 4 & 1,4-dioxane- $\mathrm{H}_{2} \mathrm{O} 5-1$ & 44 & 32 & - \\
\hline 5 & $\mathrm{CH}_{3} \mathrm{COOEt}$ & 63 & 11 & - \\
\hline 6 & Diethylcarbonate & 68 & 9 & - \\
\hline 7 & Acetone & 53 & 7 & - \\
\hline 8 & Acetone- $\mathrm{H}_{2} \mathrm{O} 9-1$ & 39 & 7 & - \\
\hline 9 & $\mathrm{MeOH}$ & 40 & 9 & - \\
\hline 10 & $\mathrm{MeOH}-\mathrm{H}_{2} \mathrm{O} 9-1$ & 29 & 9 & - \\
\hline 11 & $\mathrm{MeOH}-\mathrm{H}_{2} \mathrm{O}$ 5-1 & 33 & 10 & - \\
\hline 12 & $\mathrm{CF}_{3} \mathrm{CH}_{2} \mathrm{OH}$ & 18 & 15 & $-a$ \\
\hline 13 & $\mathrm{MeCN}$ & 46 & 9 & - \\
\hline 14 & $\mathrm{MeCN}-\mathrm{H}_{2} \mathrm{O} 9-1$ & 61 & - & - \\
\hline 15 & $\mathrm{MeCN}-\mathrm{H}_{2} \mathrm{O}$ 5-1 & 28 & 7 & - \\
\hline 16 & $\mathrm{MeCN}-\mathrm{H}_{2} \mathrm{O} 9-1^{b}$ & 56 & - & - \\
\hline 17 & $\mathrm{MeCN}-\mathrm{H}_{2} \mathrm{O} 9-1^{c}$ & 78 & - & $9 a, 6$ \\
\hline 18 & $\mathrm{MeCN}-\mathrm{H}_{2} \mathrm{O} 9-1^{d}$ & 70 & - & $9 a, 7$ \\
\hline
\end{tabular}

${ }^{a} 4$-Fluorobenzonitrile formed in $12 \%$ yield. ${ }^{b}$ Reaction carried out by exposing the reaction vessel under natural sunlight ( 3 days, 8 hours a day). ${ }^{c} \mathrm{~A} 0.1 \mathrm{M}$ solution of $\mathbf{1 a}$ in the presence of furan (2 M). ${ }^{d} \mathrm{~A} 0.1 \mathrm{M}$ solution of $\mathbf{1 a}$ in the presence of furan $(1 \mathrm{M})$. 
2. Copy of ${ }^{1} \mathrm{H}$ and ${ }^{13} \mathrm{C}$ NMR of compounds 1a-h, 2a-h, 4-8.

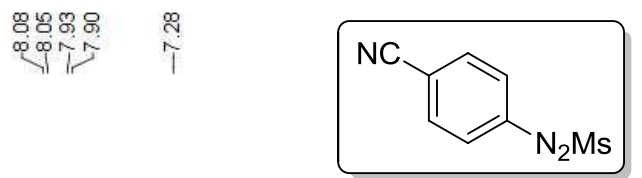

1a, ${ }^{1} \mathrm{H} \mathrm{NMR}, 300 \mathrm{MHz}, \mathrm{CDCl}_{3}$

$\stackrel{\infty}{i}$

$\iint$
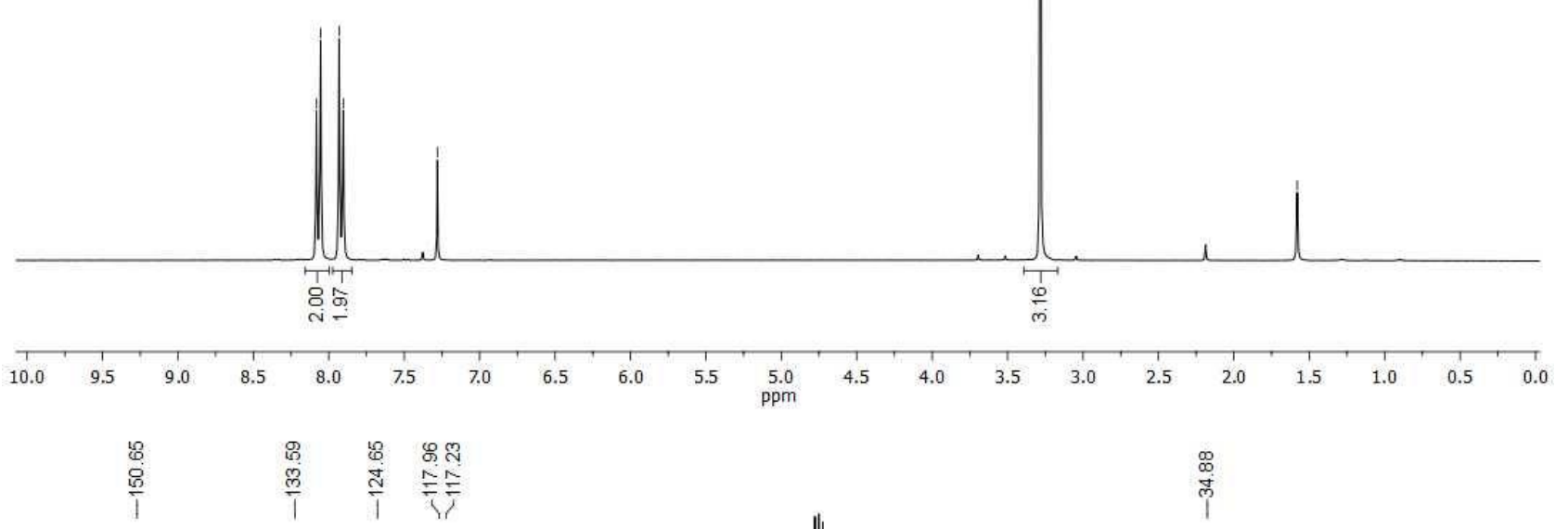

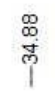

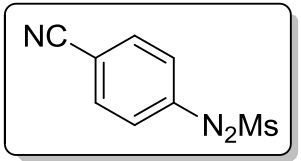

1a, ${ }^{13} \mathrm{C} \mathrm{NMR,} 75 \mathrm{MHz}, \mathrm{CDCl}_{3}$

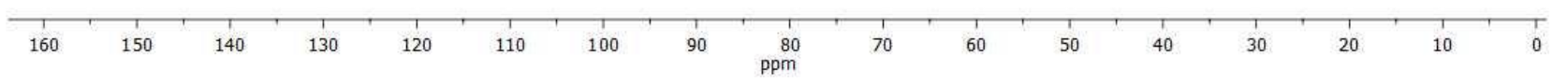

S9 


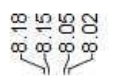

$\iint$

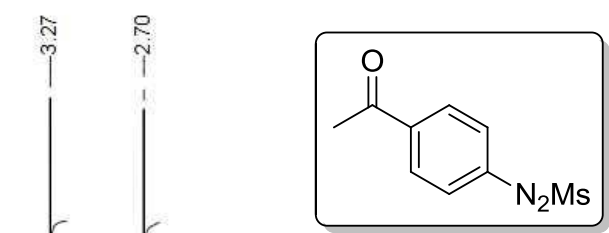

1b, ${ }^{1} \mathrm{H} \mathrm{NMR}, 300 \mathrm{MHz}, \mathrm{CDCl}_{3}$
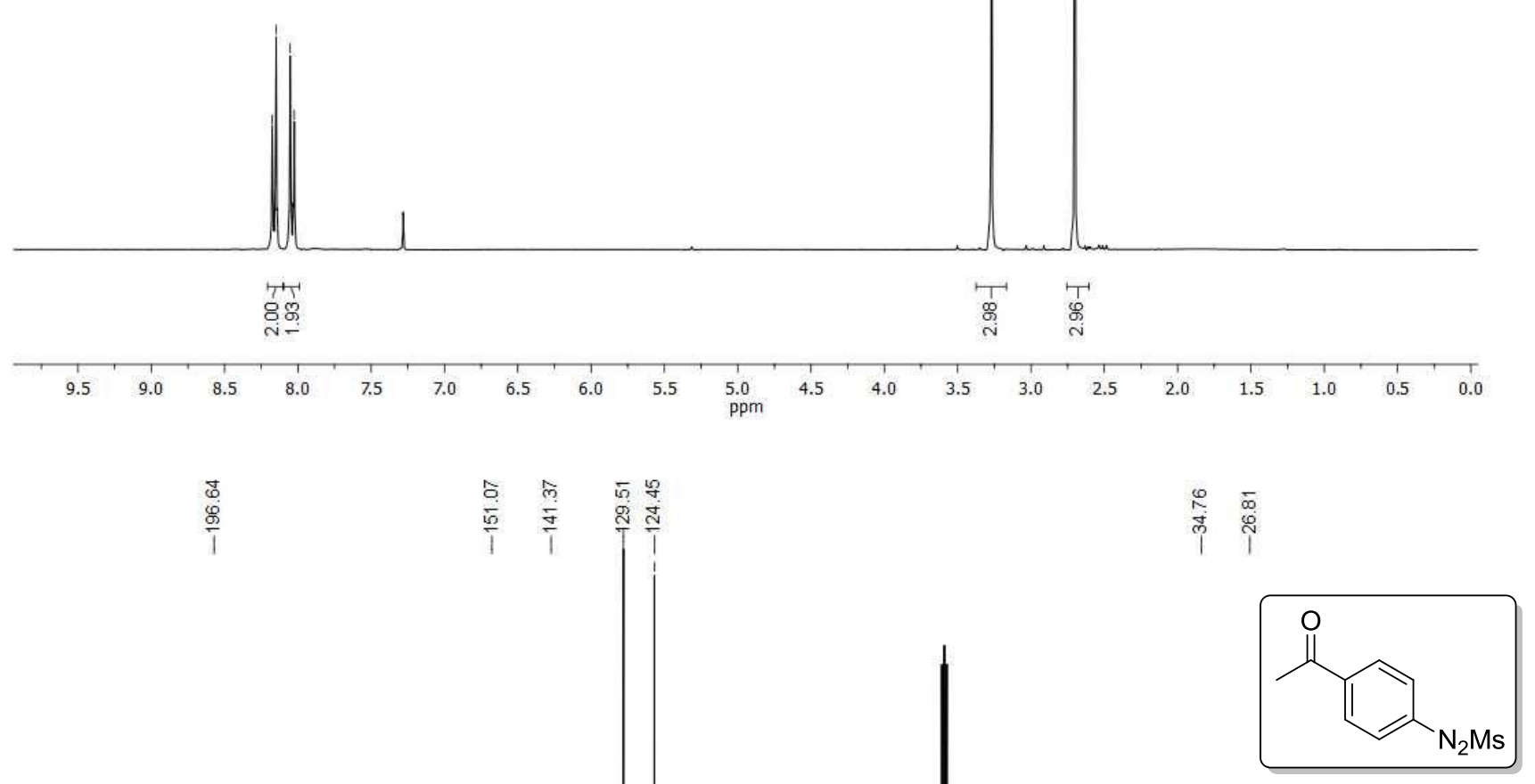

1b, ${ }^{13} \mathrm{C} \mathrm{NMR}, 75 \mathrm{MHz}, \mathrm{CDCl}_{3}$

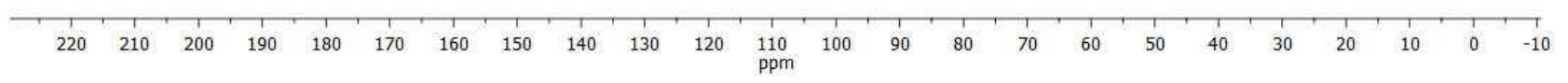

S10 

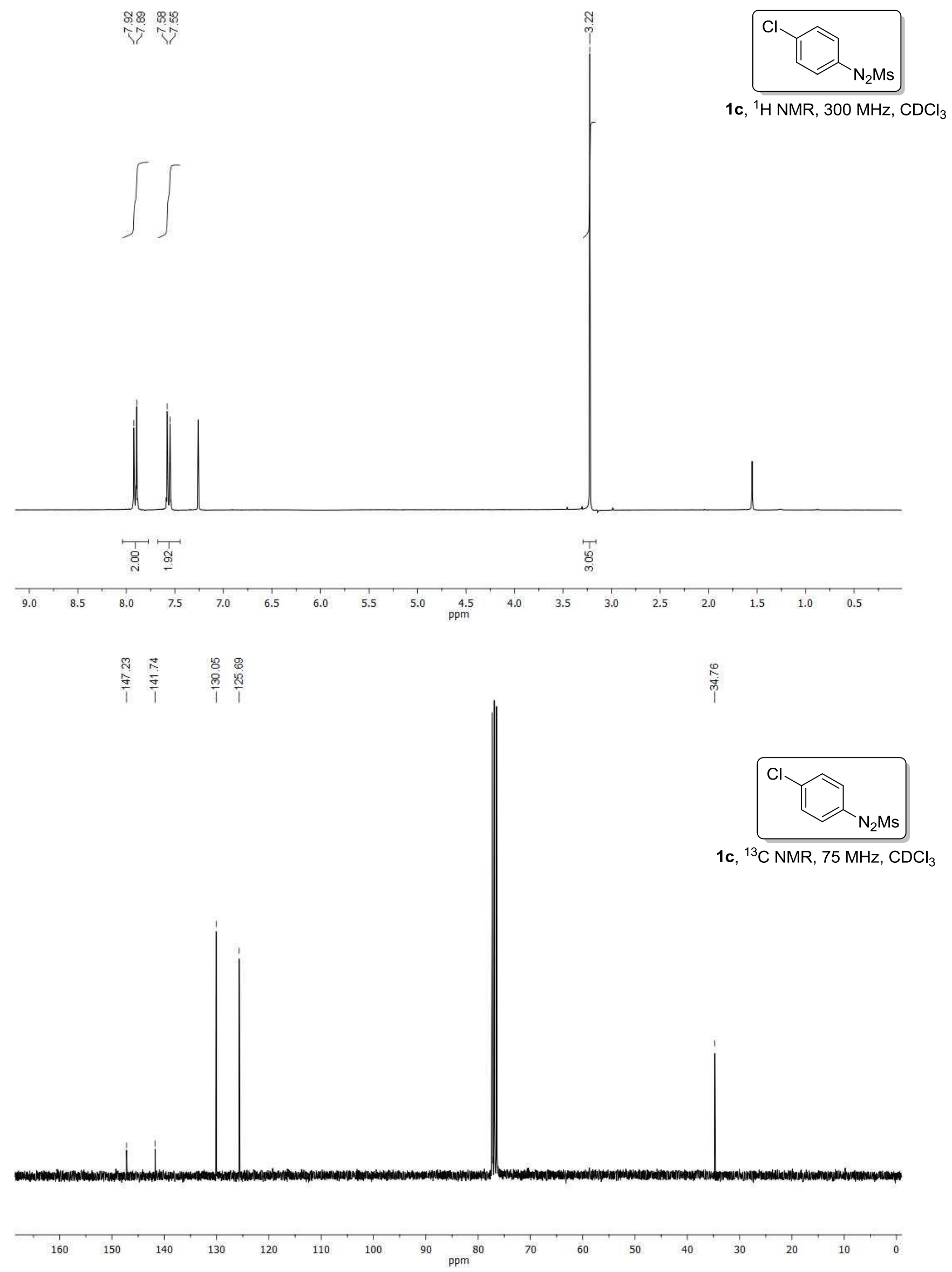


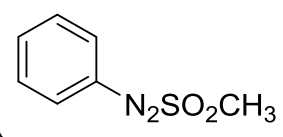

1d, ${ }^{1} \mathrm{H} \mathrm{NMR}, 300 \mathrm{MHz}, \mathrm{CDCl}_{3}$
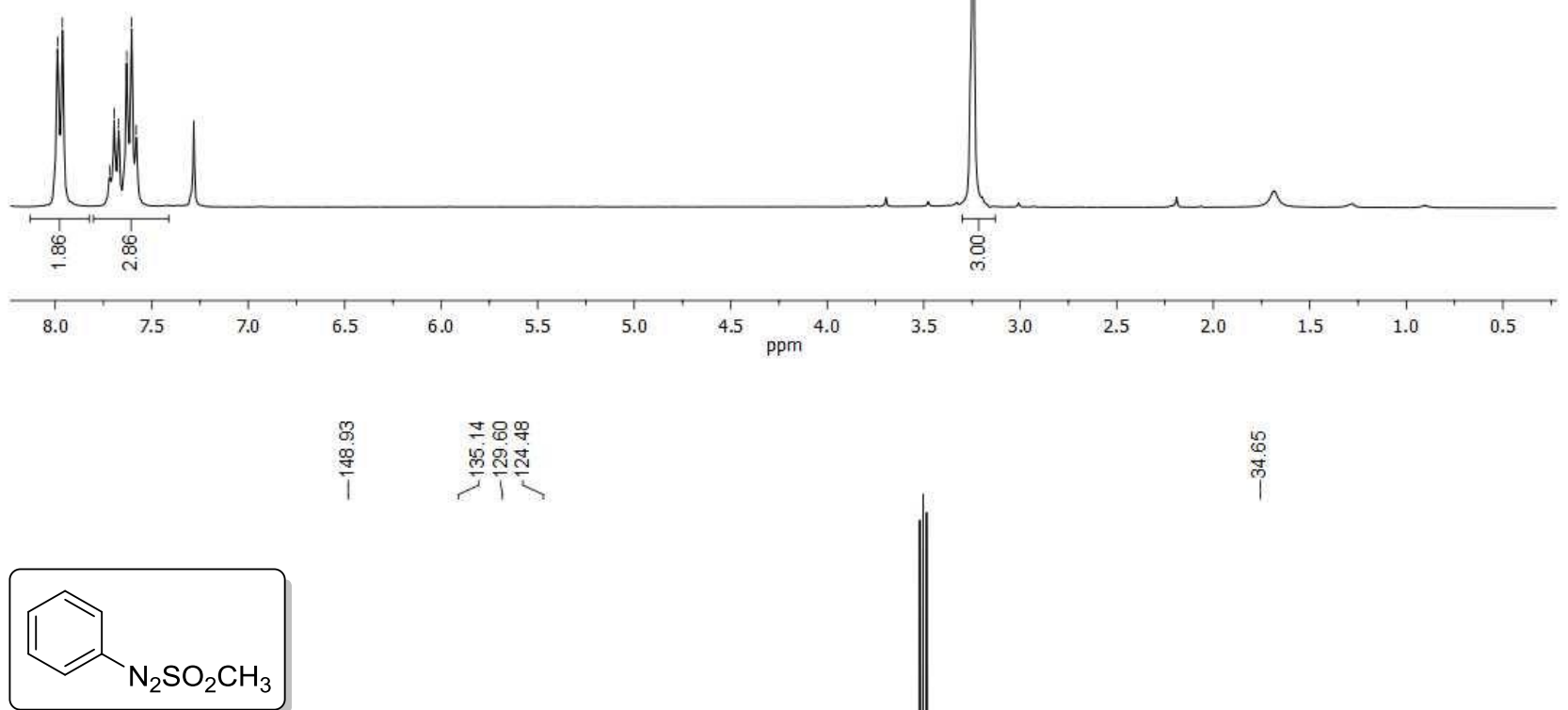

1d, ${ }^{13} \mathrm{C}$ NMR, $75 \mathrm{MHz}, \mathrm{CDCl}_{3}$

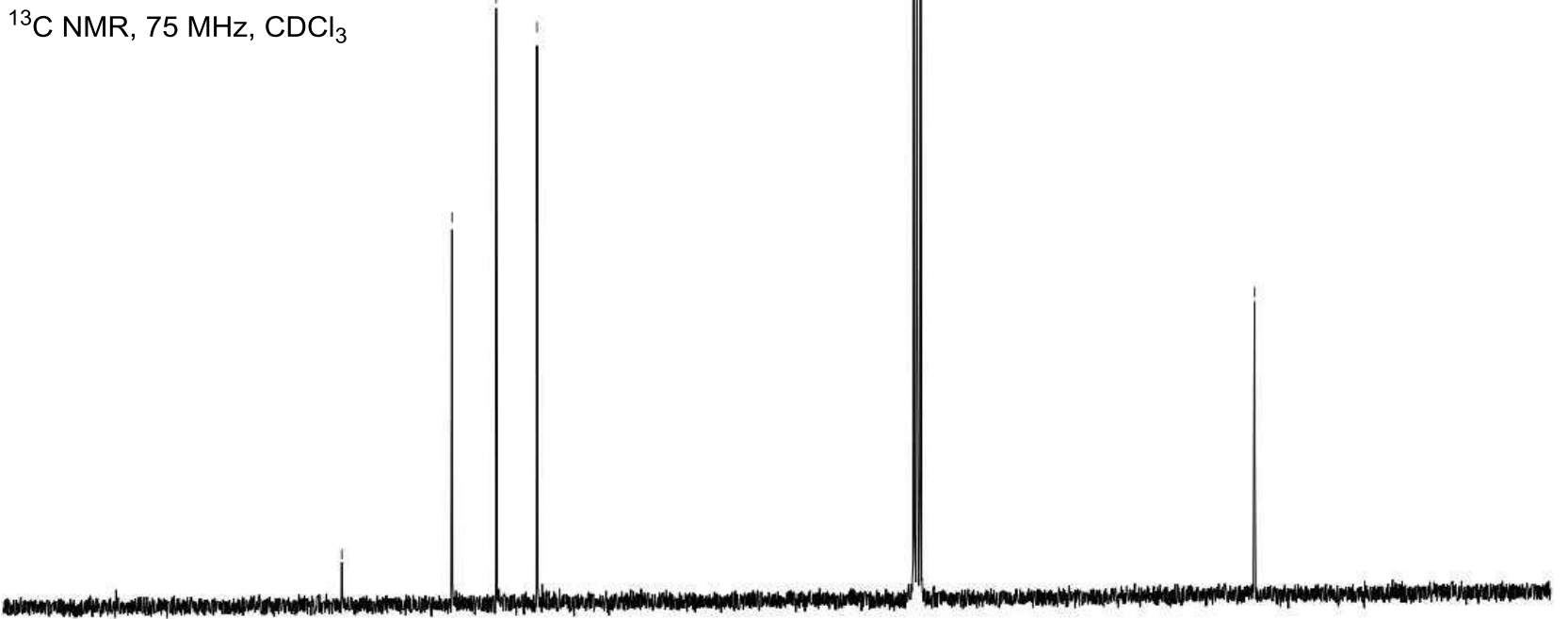




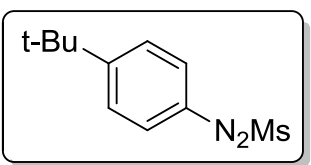

$\stackrel{\text { กิ }}{\text { i }}$

1e, ${ }^{1} \mathrm{H} \mathrm{NMR}, 300 \mathrm{MHz}, \mathrm{CDCl}_{3}$

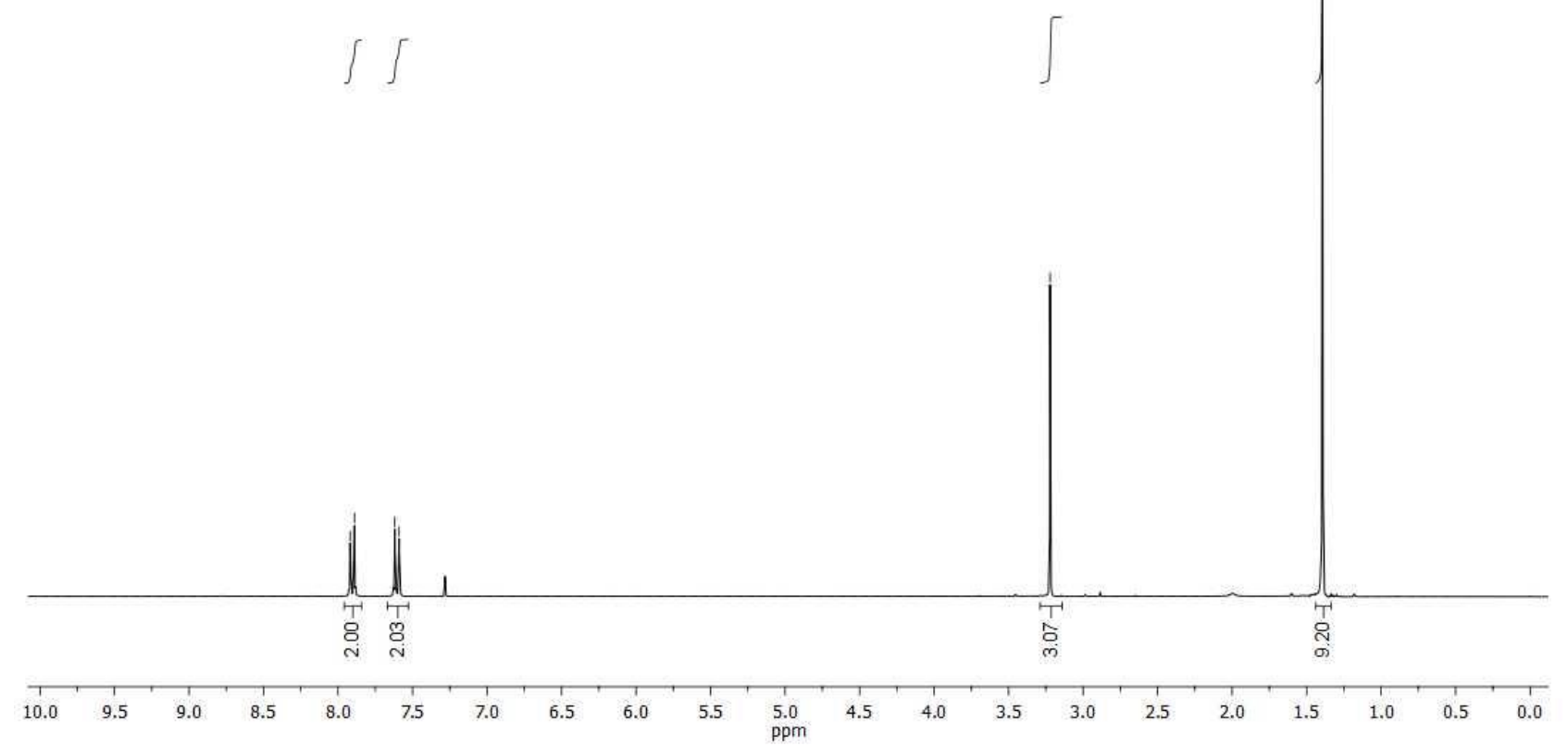

$\begin{array}{lll}\frac{1}{2} & \infty & 08 \\ 0 & \frac{1}{0} & \frac{1}{2}\end{array}$

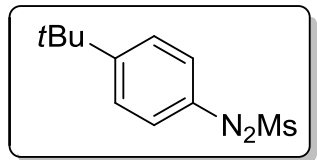

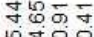

눈

1e, ${ }^{13} \mathrm{C} \mathrm{NMR}, 75 \mathrm{MHz}, \mathrm{CDCl}_{3}$
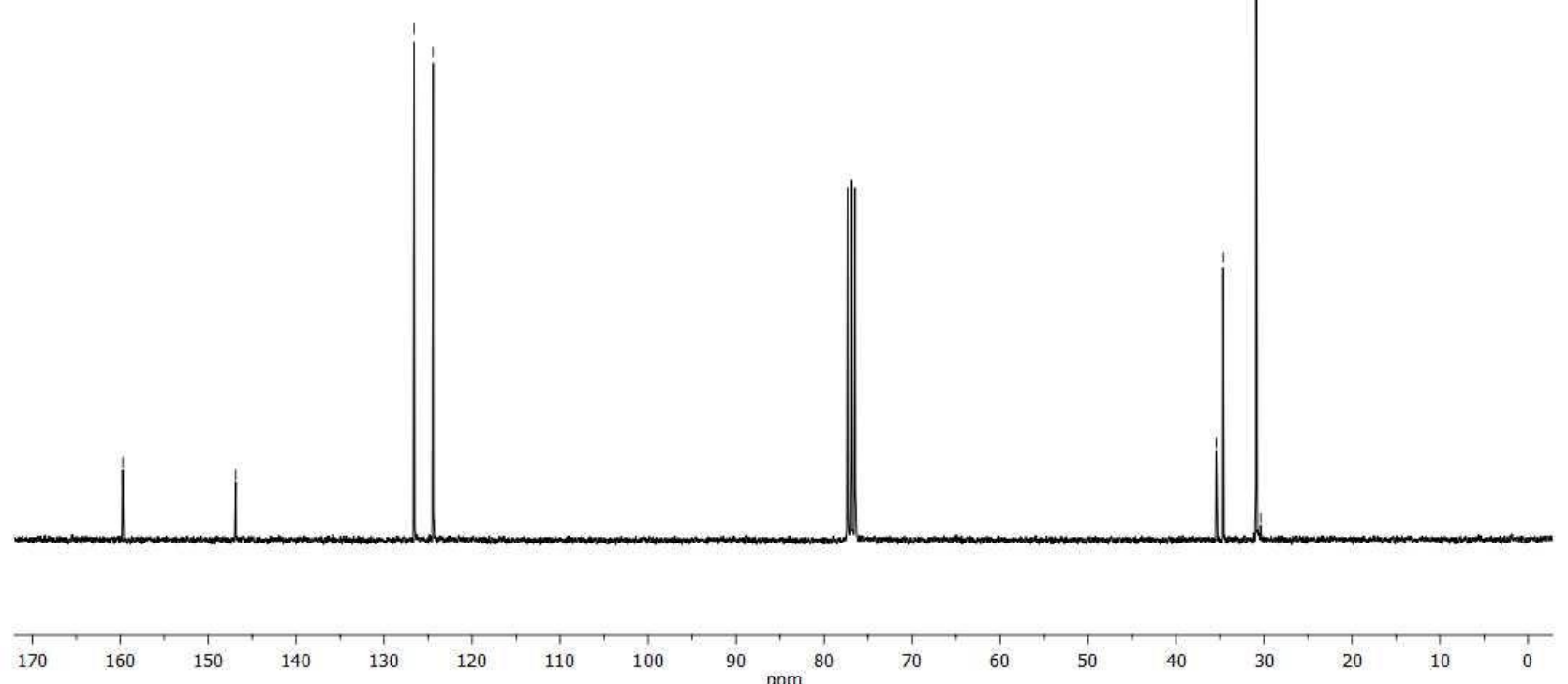

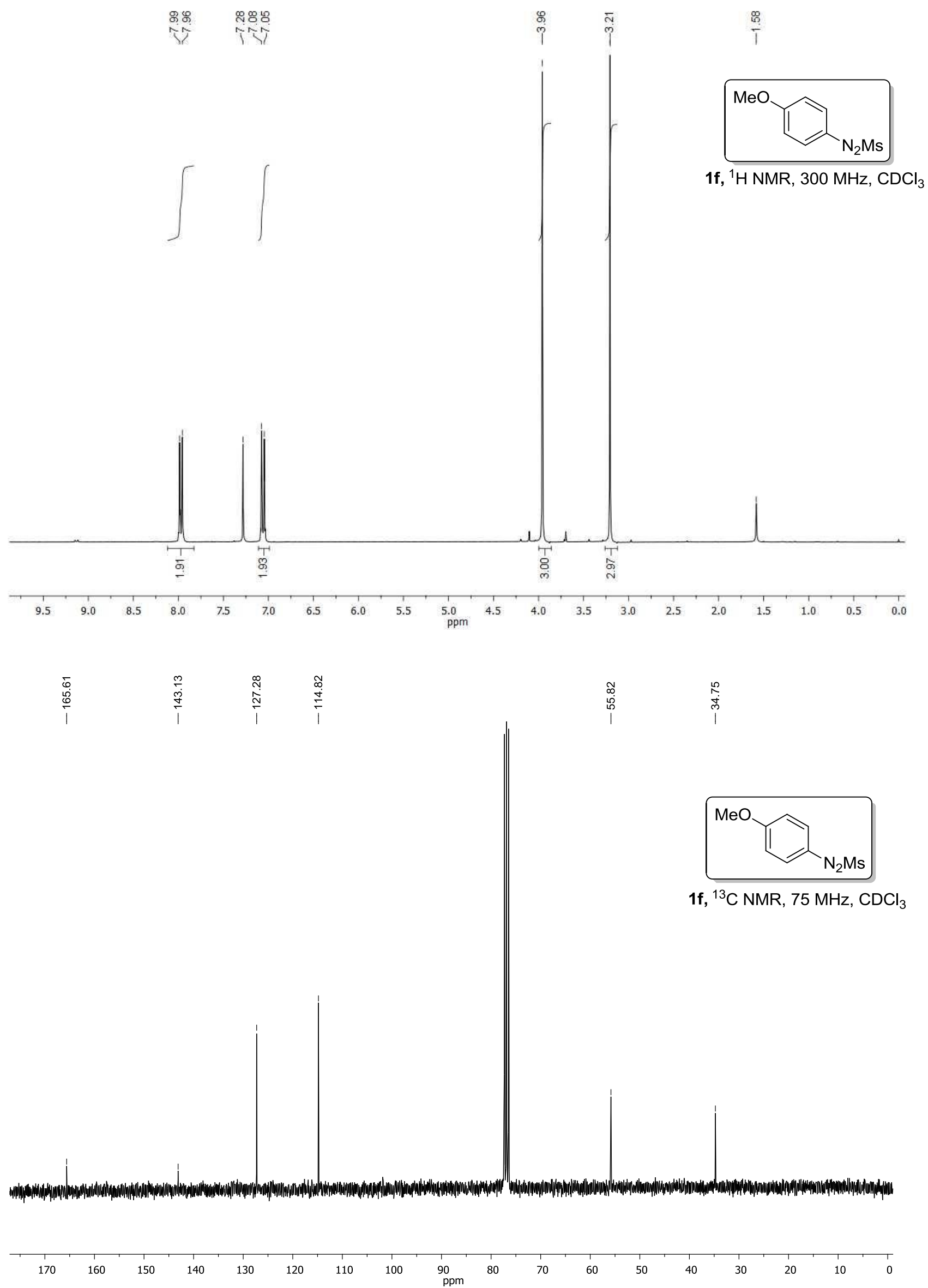


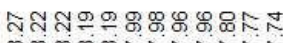

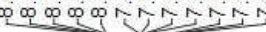

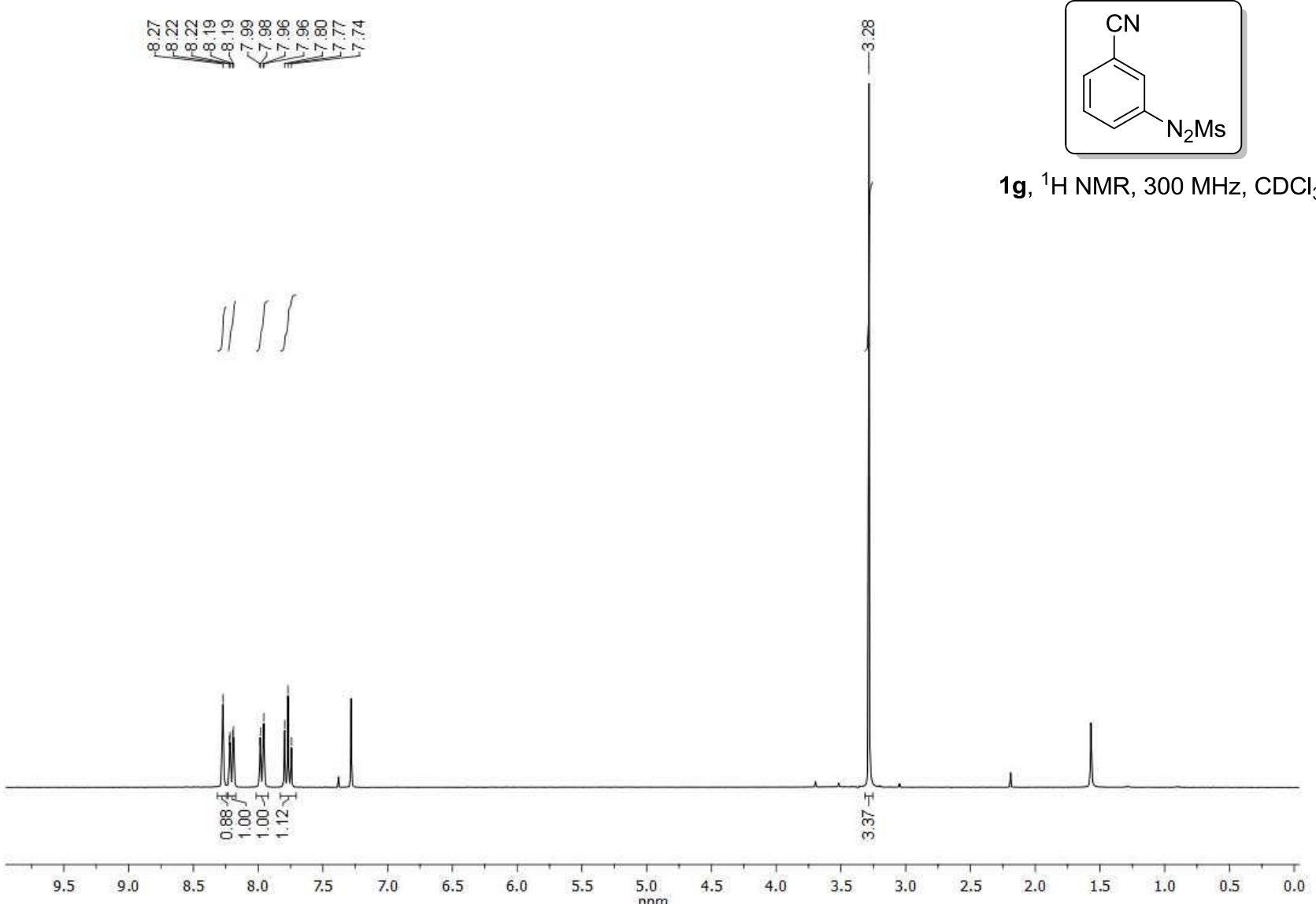

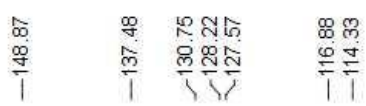

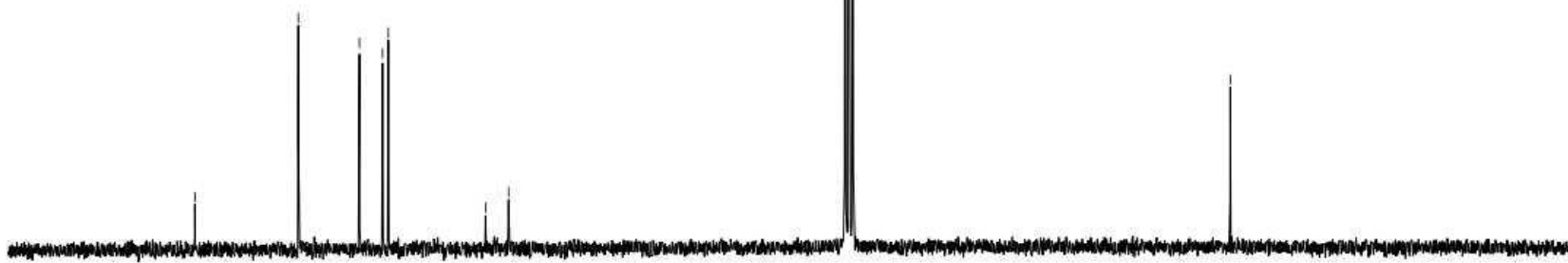

స్

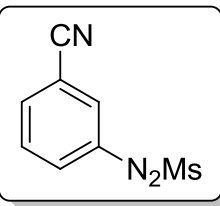

1g, ${ }^{13} \mathrm{C} \mathrm{NMR}, 75 \mathrm{MHz}, \mathrm{CDCl}_{3}$

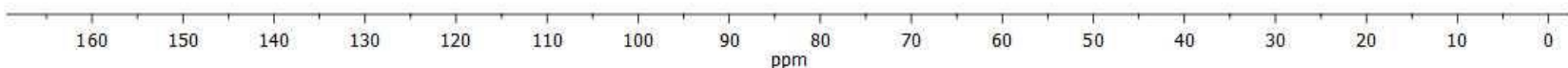




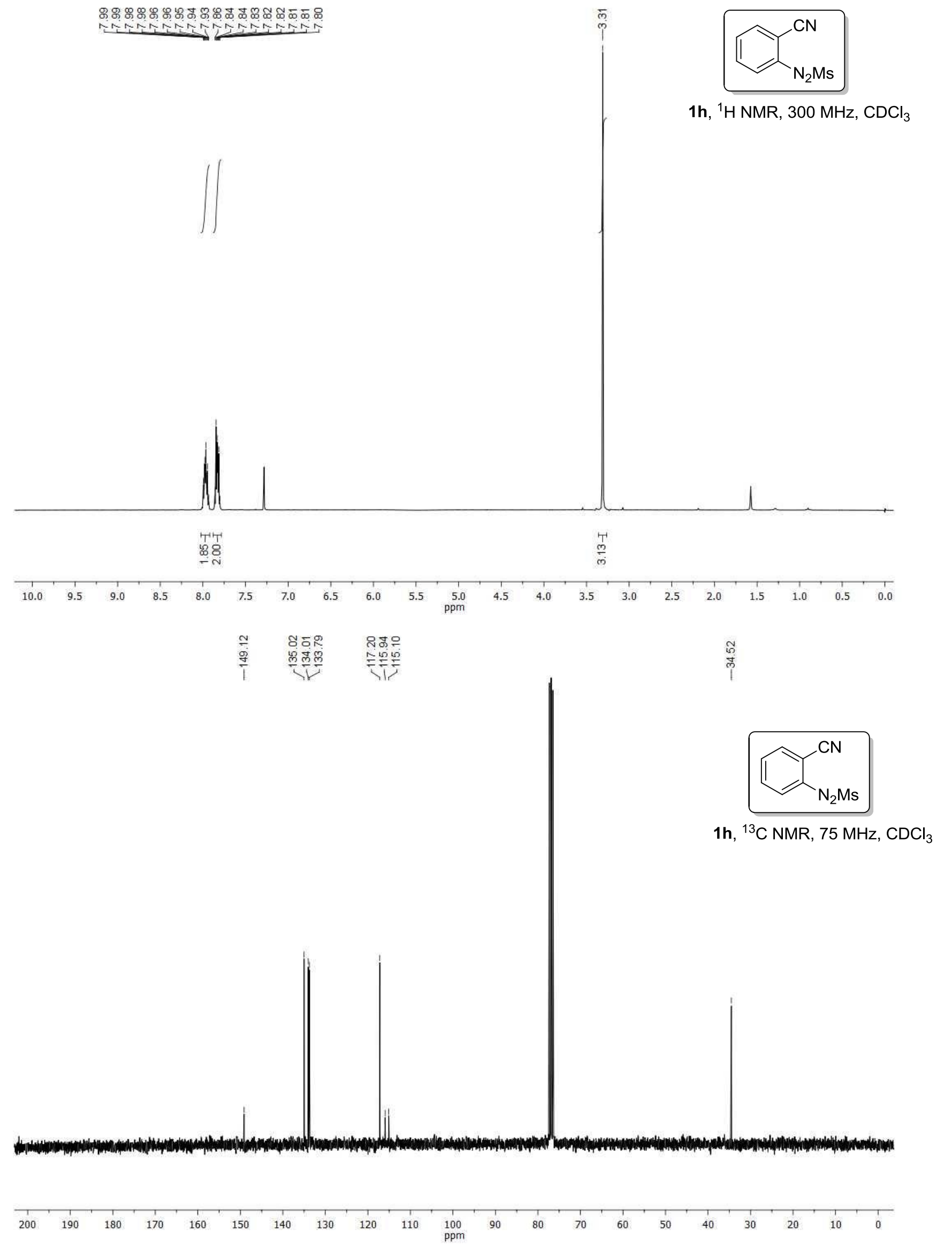



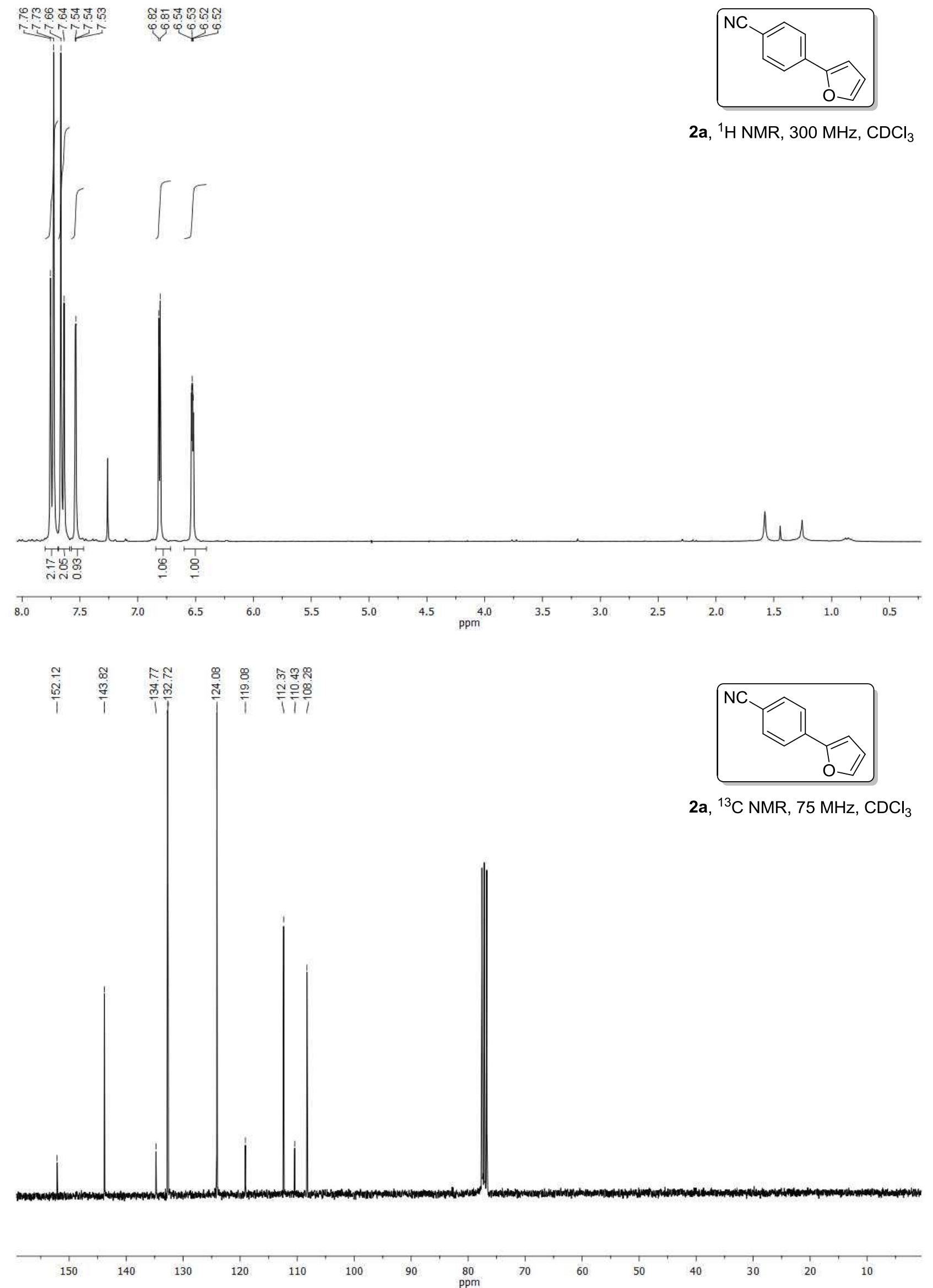

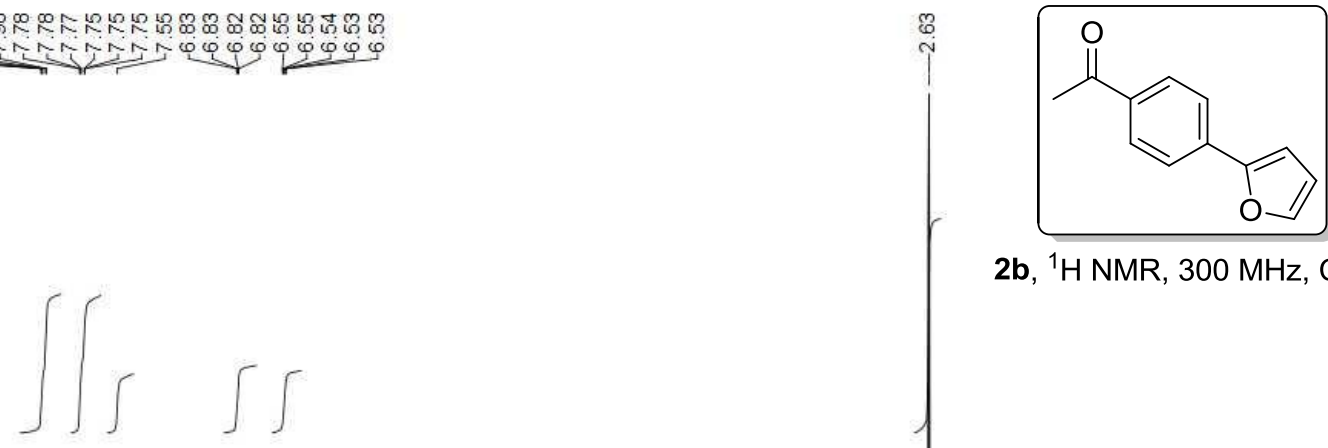

2b, ${ }^{1} \mathrm{H}$ NMR, $300 \mathrm{MHz}, \mathrm{CDCl}_{3}$
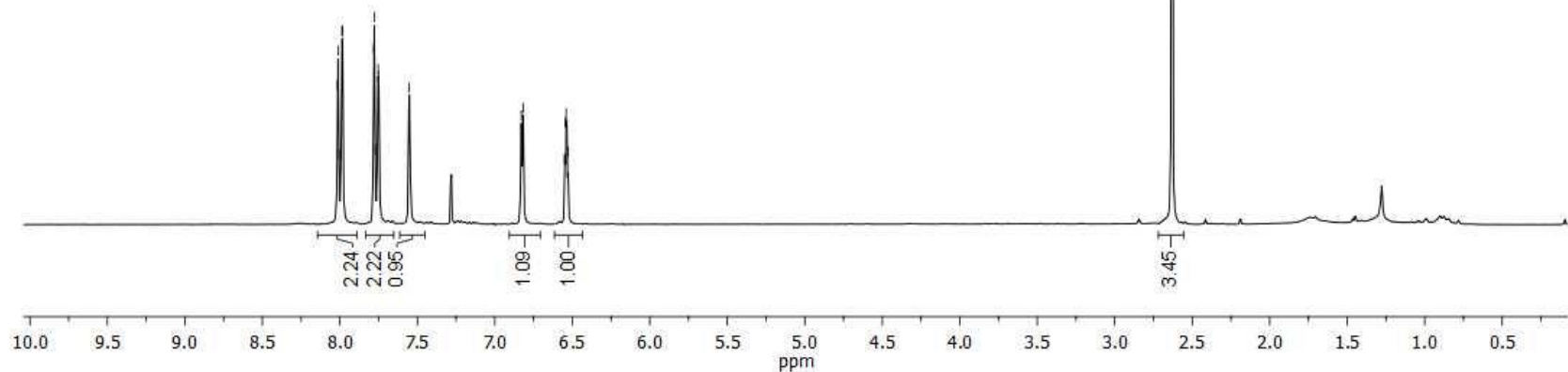

ํํํ

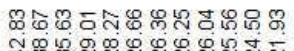

ํํำ

ขे
ขั

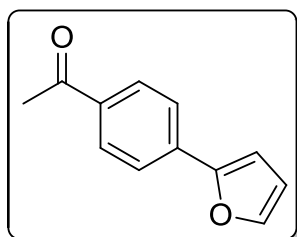

2b, ${ }^{13} \mathrm{C} \mathrm{NMR}, 75 \mathrm{MHz}, \mathrm{CDCl}_{3}$

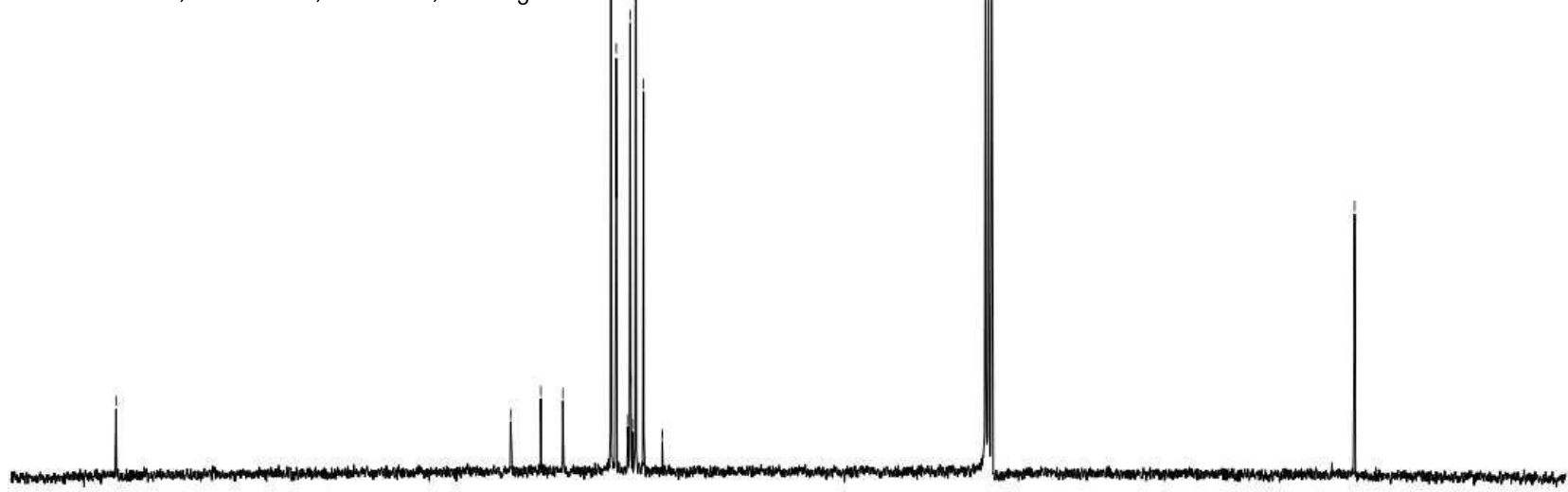

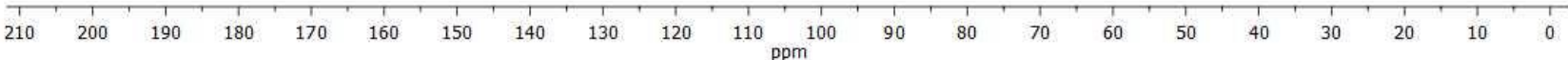



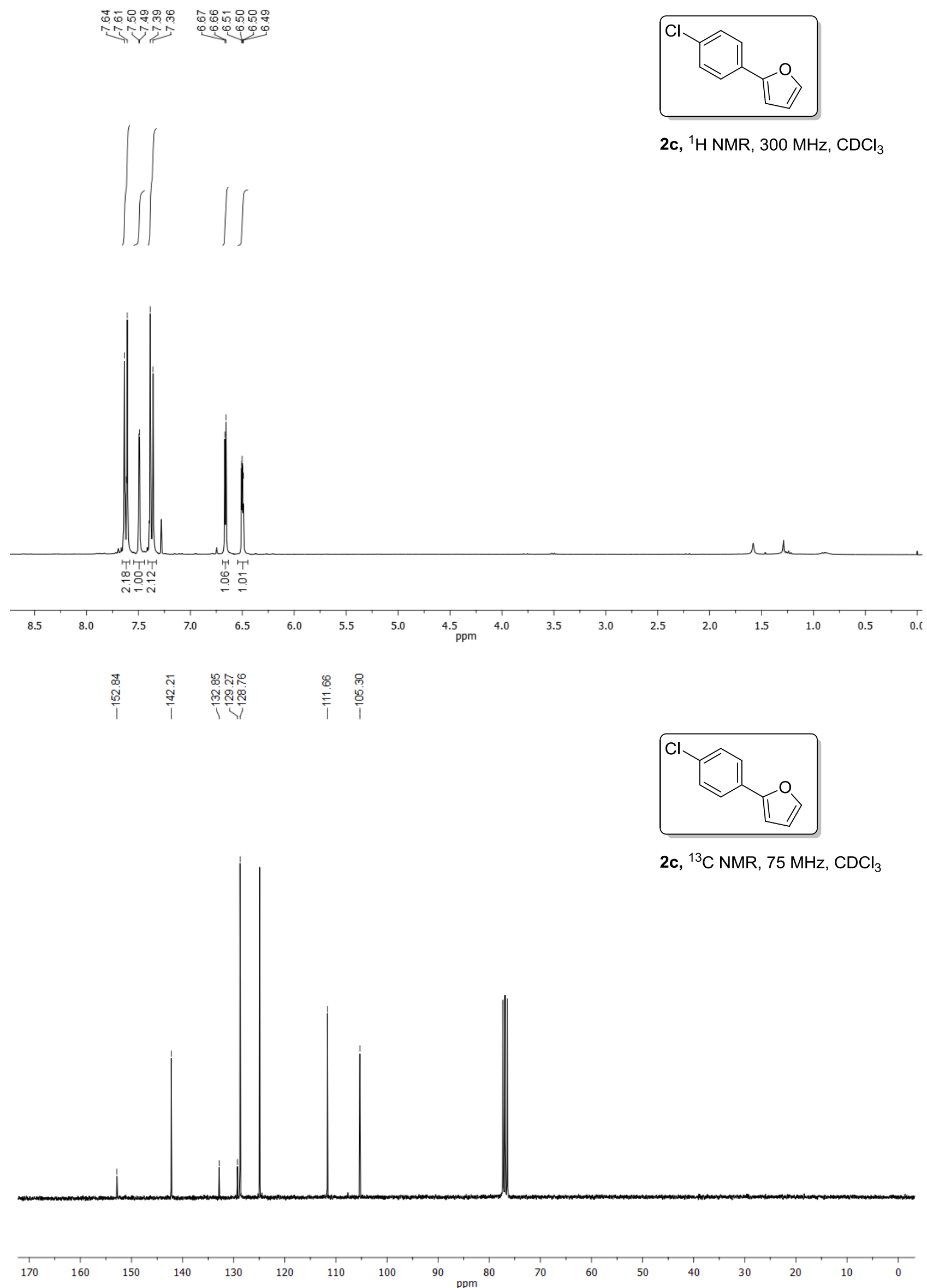


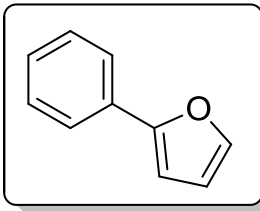

2d, ${ }^{1} \mathrm{H}$ NMR, $300 \mathrm{MHz}, \mathrm{CDCl}_{3}$

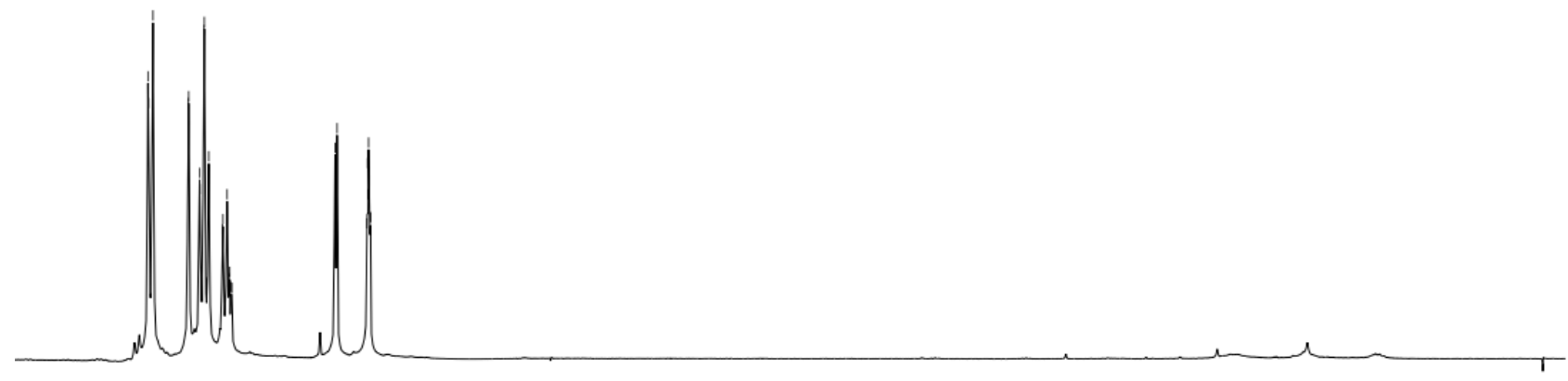

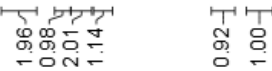
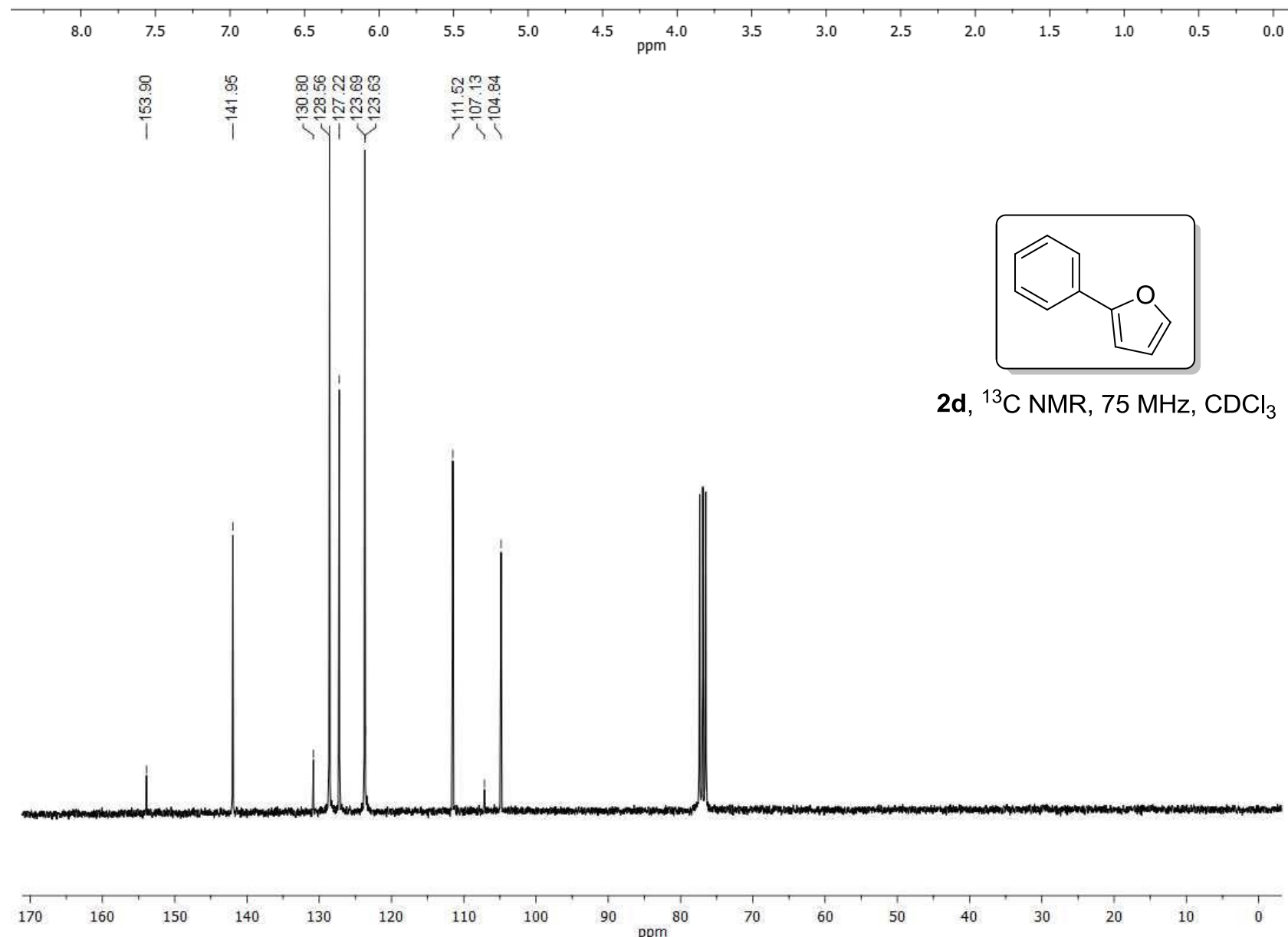


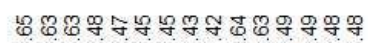

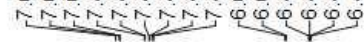

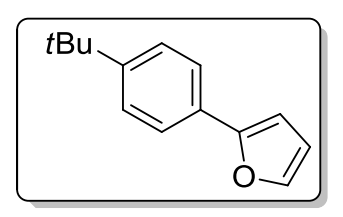

2e, ${ }^{1} \mathrm{H}$ NMR, $300 \mathrm{MHz}, \mathrm{CDCl}_{3}$
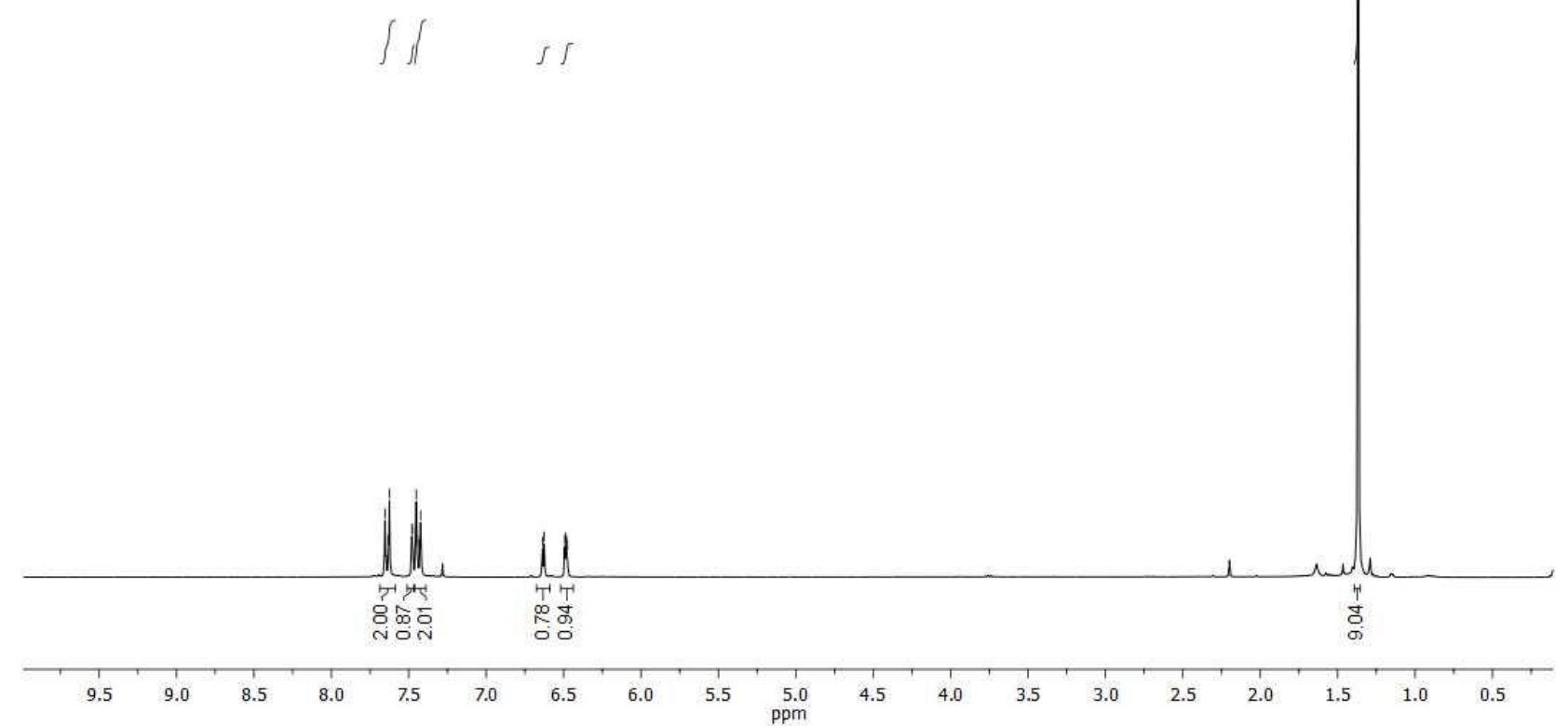

$$
\text { ๘ั }
$$

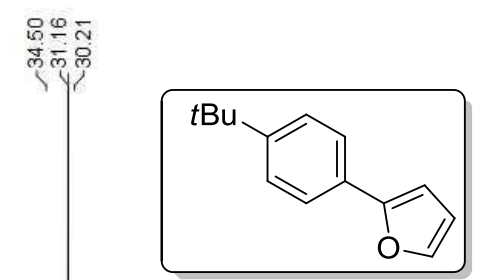

2e, ${ }^{13} \mathrm{C}$ NMR, $75 \mathrm{MHz}, \mathrm{CDCl}_{3}$

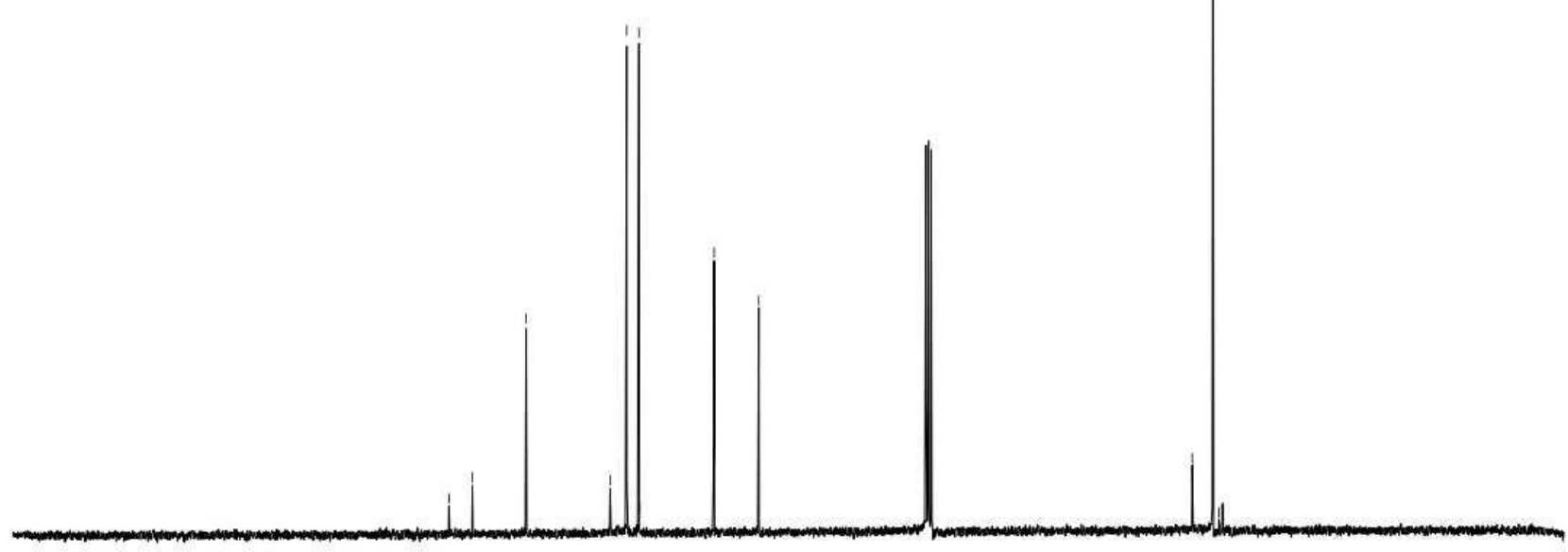

\begin{tabular}{lllllllllllllllllllllllllllll}
\hline 220 & 210 & 200 & 190 & 180 & 170 & 160 & 150 & 140 & 130 & 120 & 110 & 100 & 90 & 80 & 70 & 60 & 50 & 40 & 30 & 20 & 10 & 0 & -10 & -20
\end{tabular} 

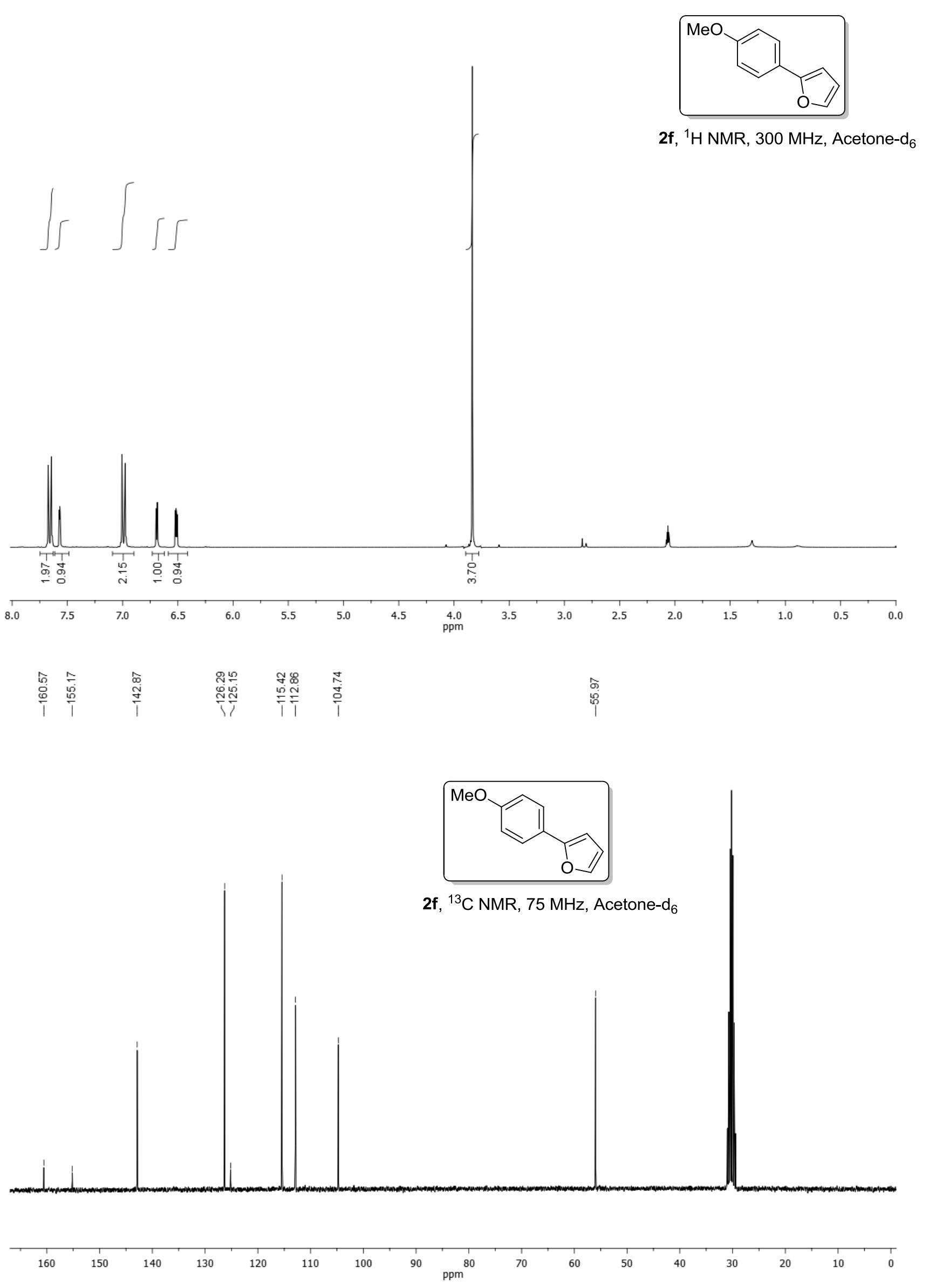

S22 

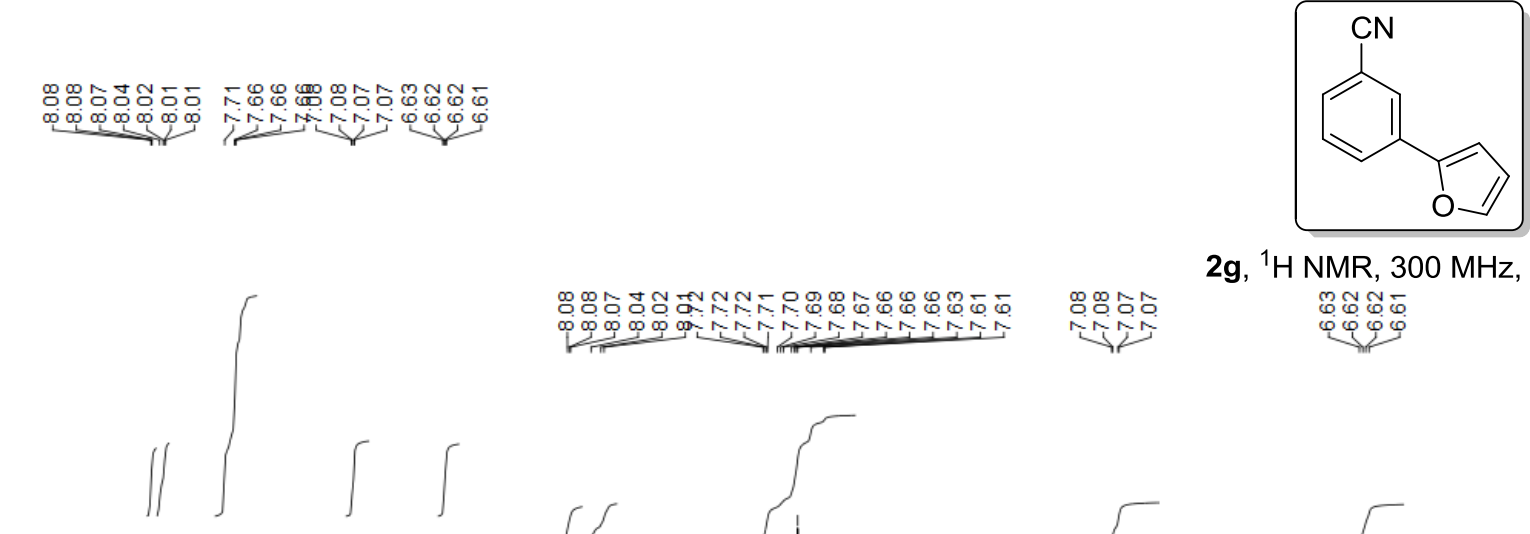

2g, ${ }^{1} \mathrm{H}$ NMR, $300 \mathrm{MHz}$, Acetone- $\mathrm{d}_{6}$

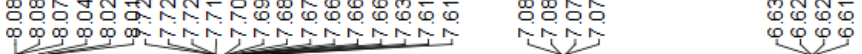
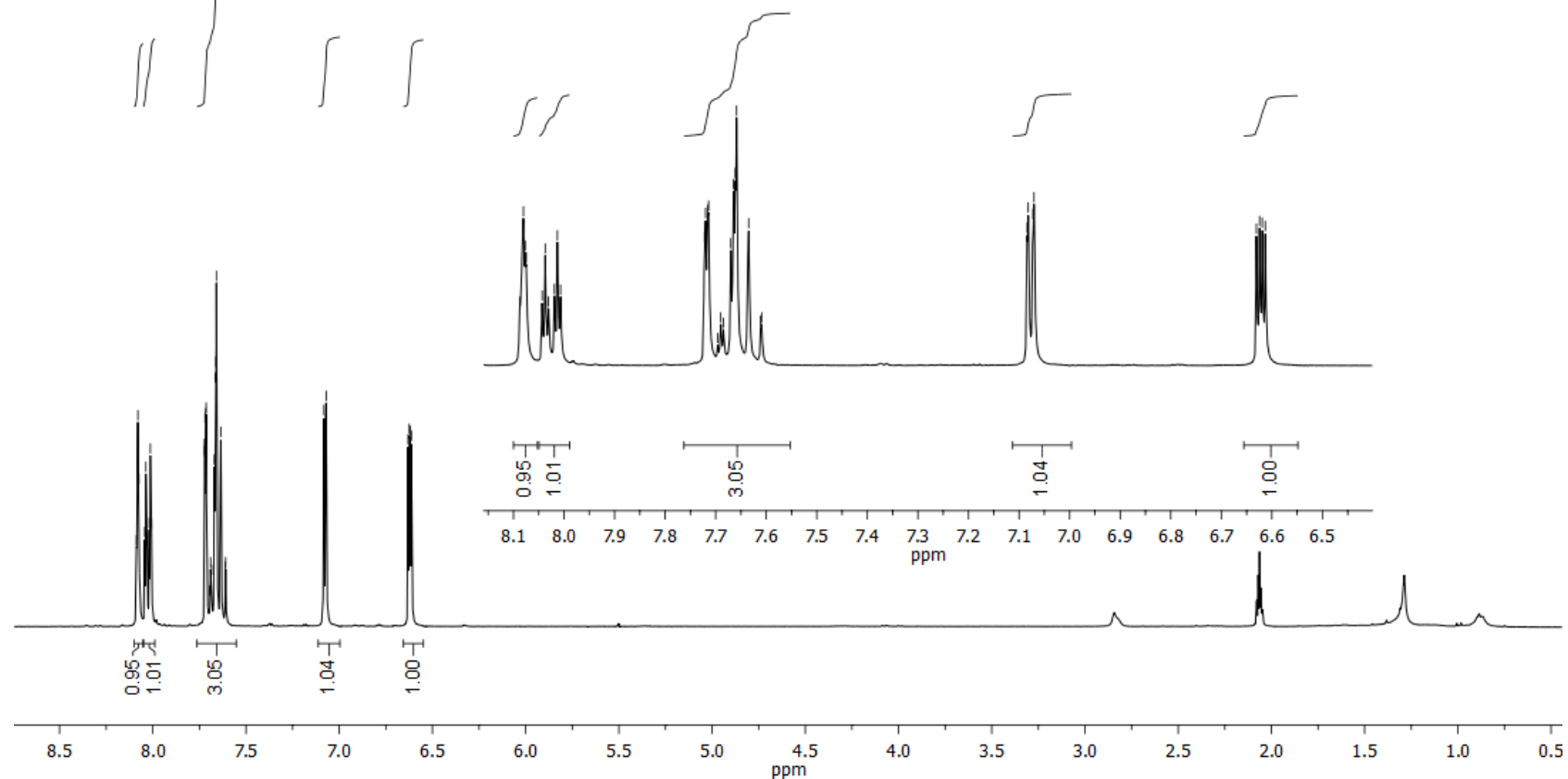

至

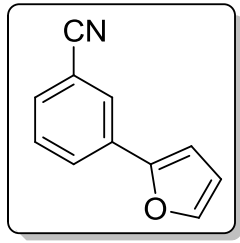

2g, ${ }^{13} \mathrm{C}$ NMR, $75 \mathrm{MHz}$, Acetone- $\mathrm{d}_{6}$

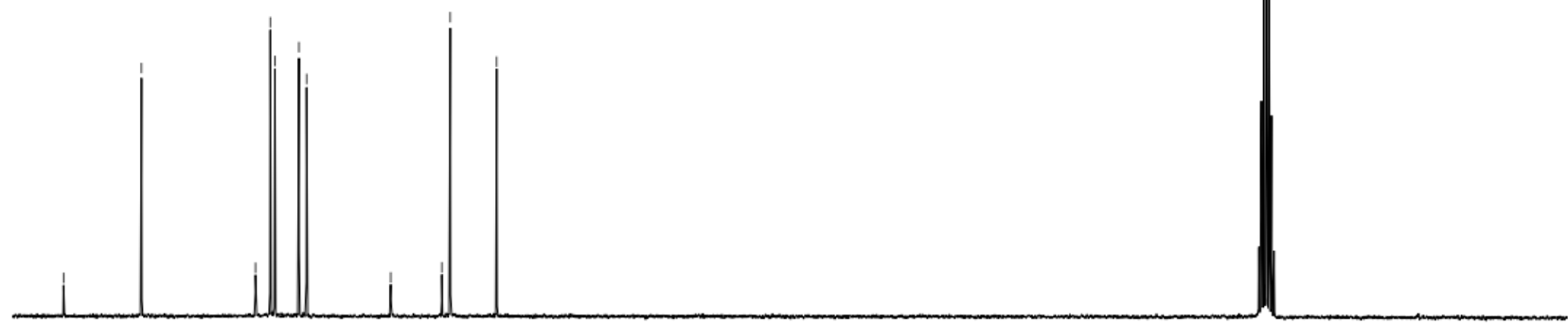

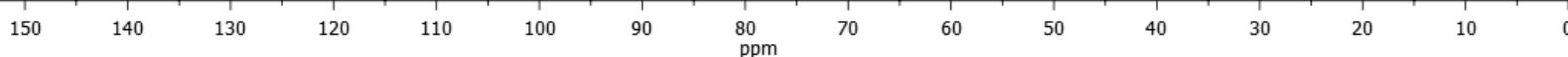



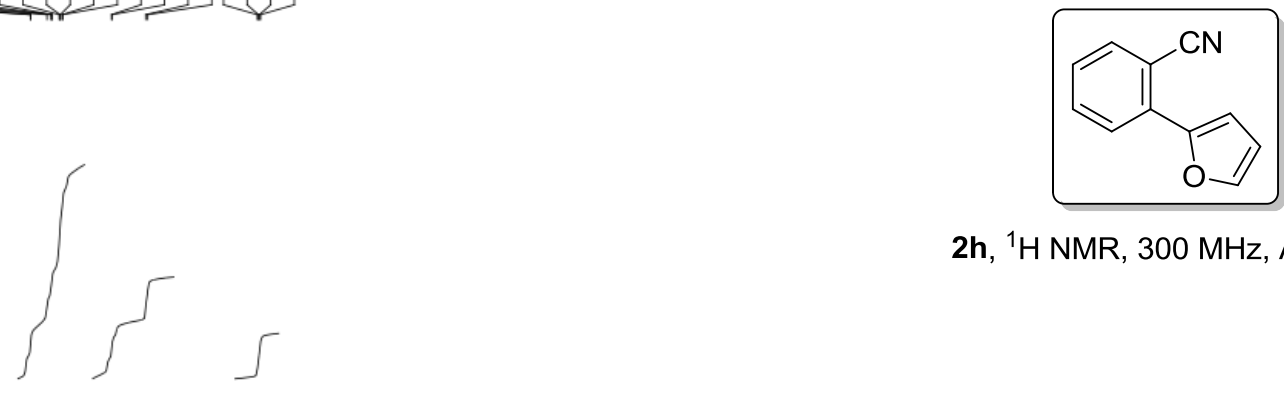

2h, ${ }^{1} \mathrm{H}$ NMR, $300 \mathrm{MHz}$, Acetone- $\mathrm{d}_{6}$

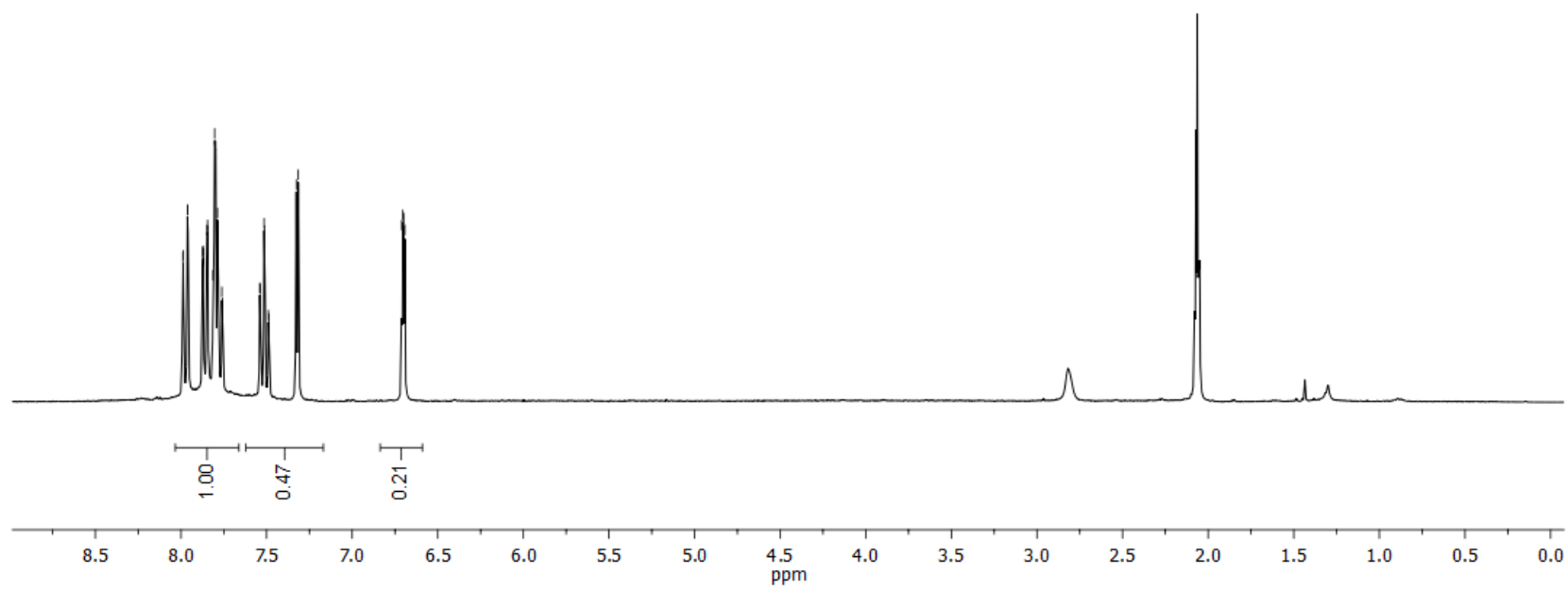

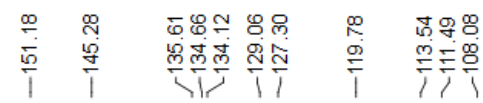

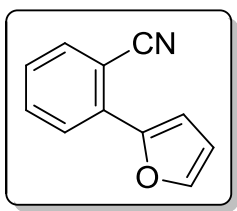

2h, ${ }^{13} \mathrm{C}$ NMR, $75 \mathrm{MHz}$, Acetone- $\mathrm{d}_{6}$
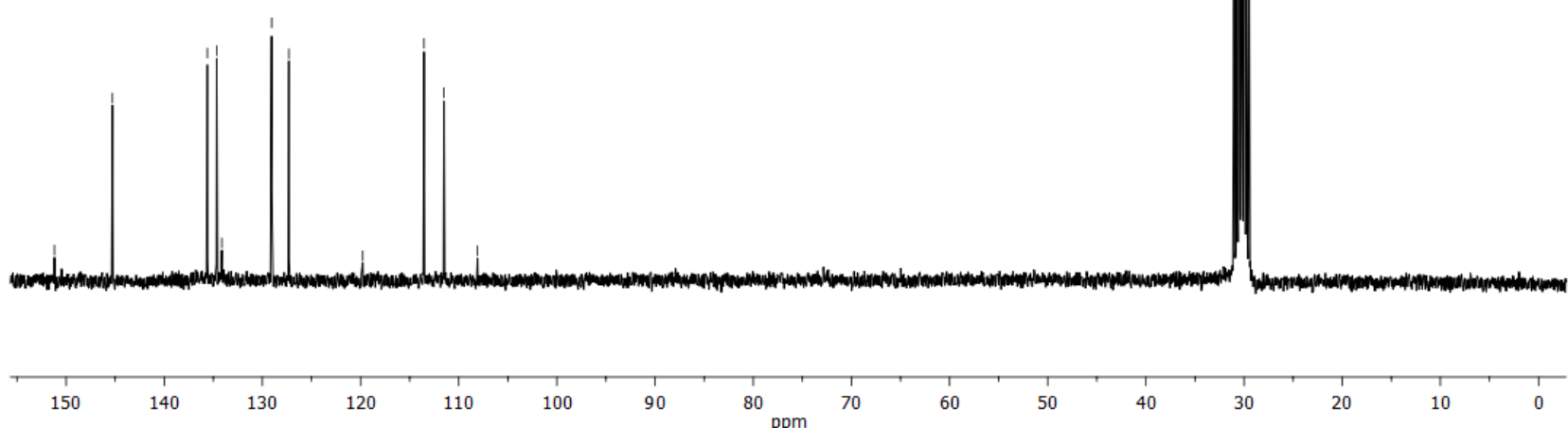


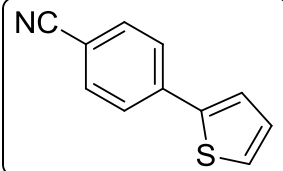

4a, ${ }^{1} \mathrm{H} \mathrm{NMR}, 300 \mathrm{MHz}, \mathrm{CDCl}_{3}$
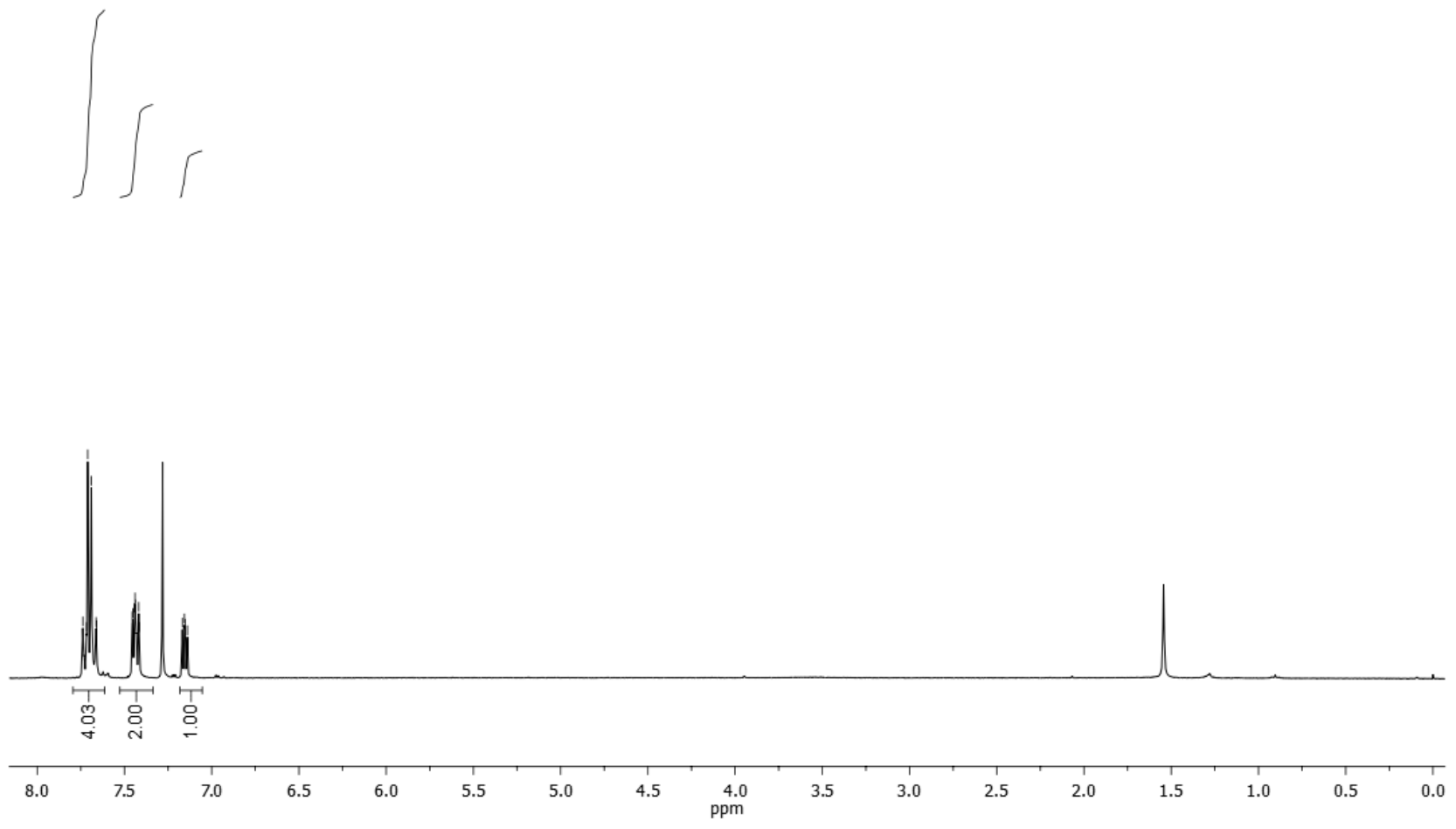

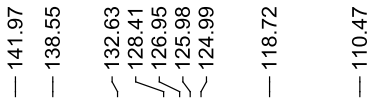

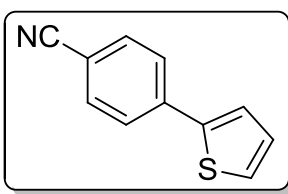

4a, ${ }^{13} \mathrm{C} \mathrm{NMR}, 75 \mathrm{MHz}, \mathrm{CDCl}_{3}$

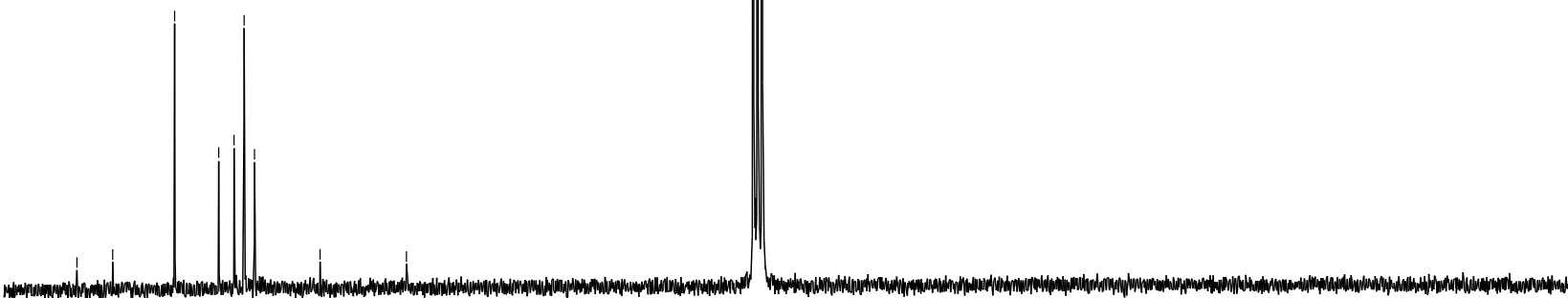

$\begin{array}{llllllllllllllllllllllllllllllll}145 & 140 & 135 & 130 & 125 & 120 & 115 & 110 & 105 & 100 & 95 & 90 & 85 & 80 & 75 & 70 & 65 & 60 & 55 & 50 & 45 & 40 & 35 & 30 & 25 & 20 & 15 & 10 & 5 & 0\end{array}$ 


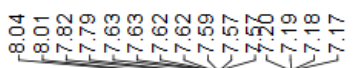

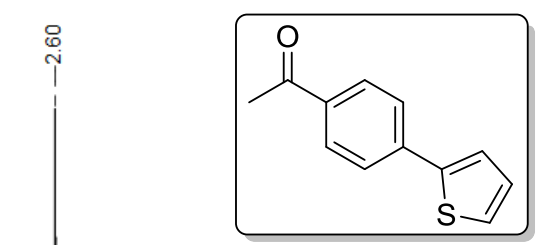

4b, ${ }^{1} \mathrm{H}$ NMR, $300 \mathrm{MHz}$, Acetone- $d_{6}$
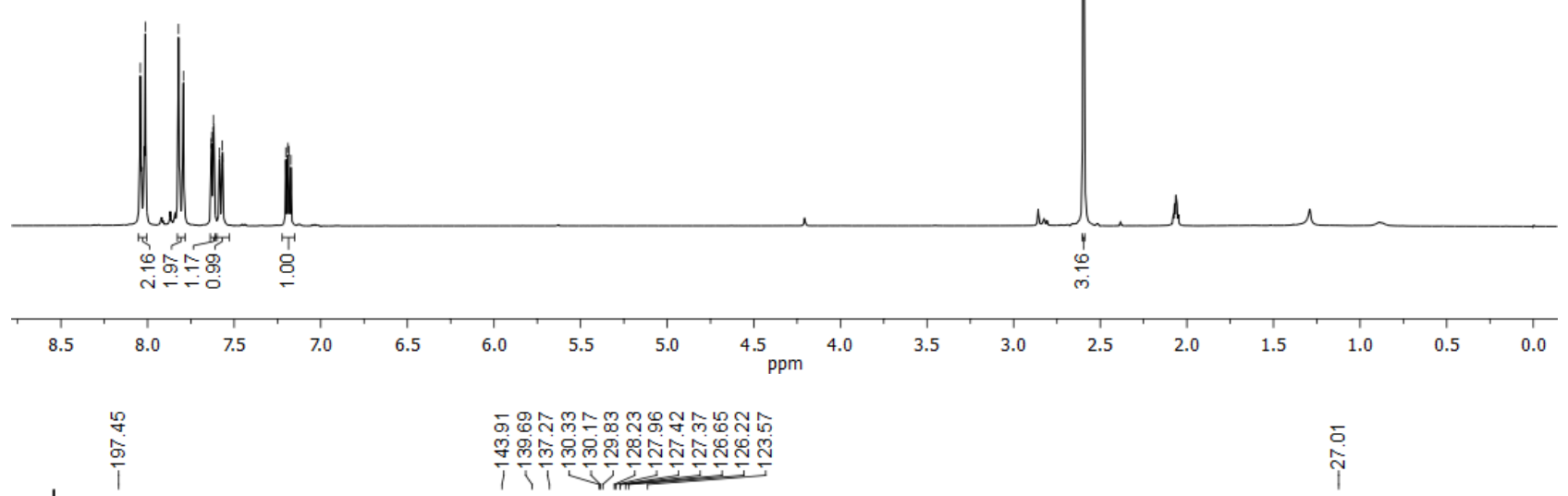

$\stackrel{\bar{c}}{i}$

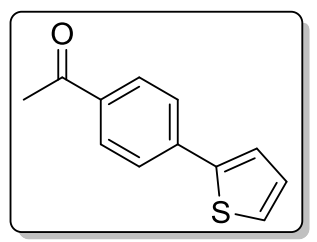

4b, ${ }^{13} \mathrm{C}$ NMR, $75 \mathrm{MHz}$, Acetone-d6

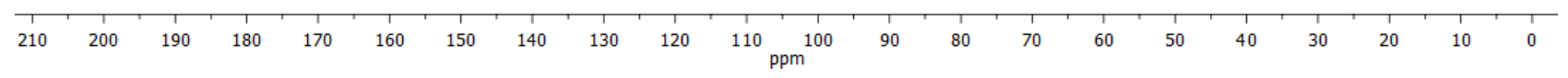

S26 


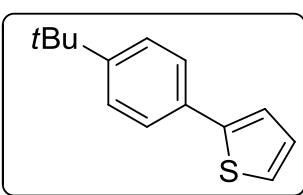

4e, ${ }^{1} \mathrm{H}$ NMR, $300 \mathrm{MHz}, \mathrm{CDCl}_{3}$

\section{1,}

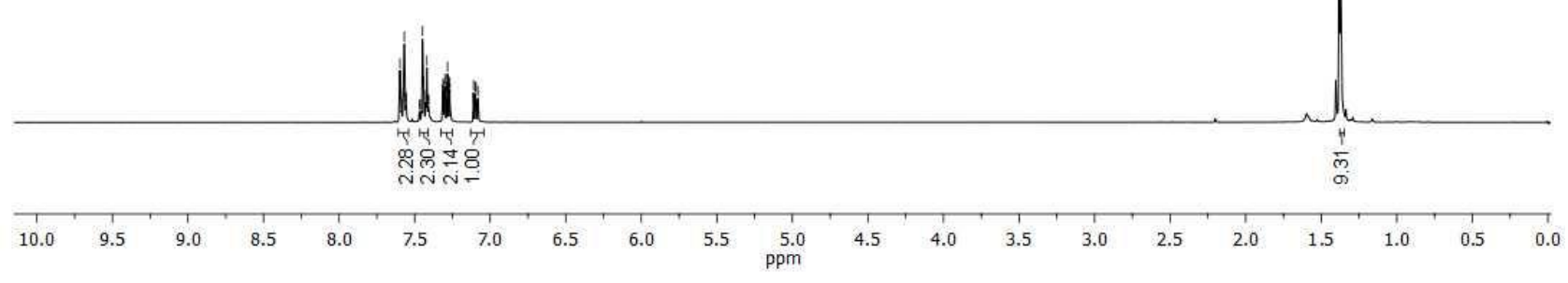

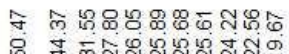

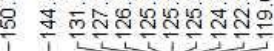

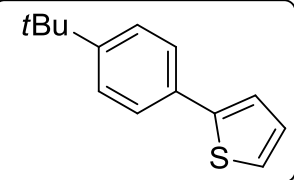

4e, ${ }^{13} \mathrm{C} \mathrm{NMR}, 75 \mathrm{MHz}, \mathrm{CDCl}_{3}$

$\begin{array}{lllllllllllll}220 & 210 & 200 & 190 & 180 & 170 & 160 & 150 & 140 & 130 & 120 & 110 & 100\end{array}$ 


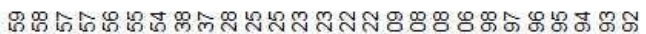

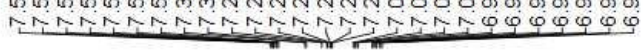
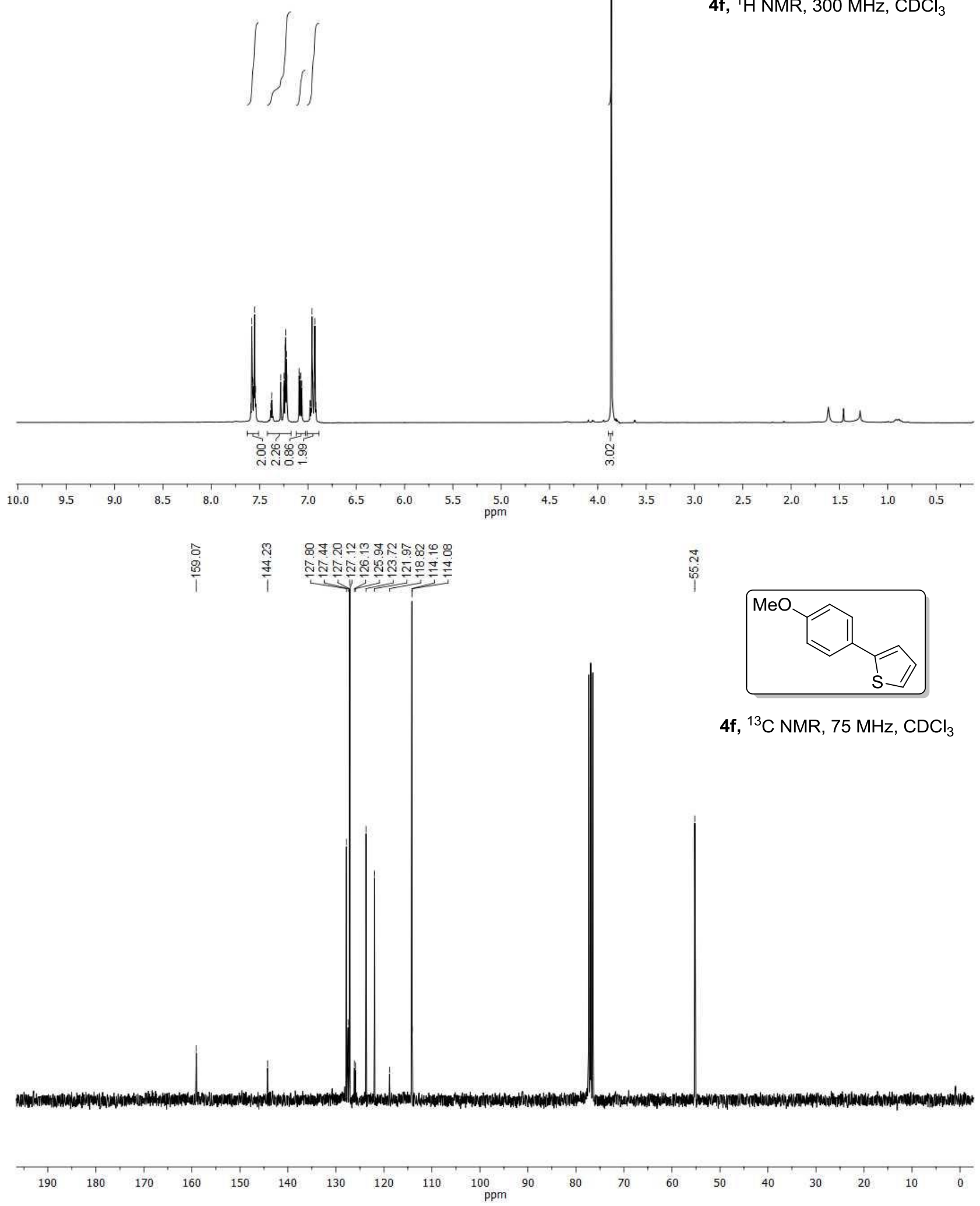

S28 

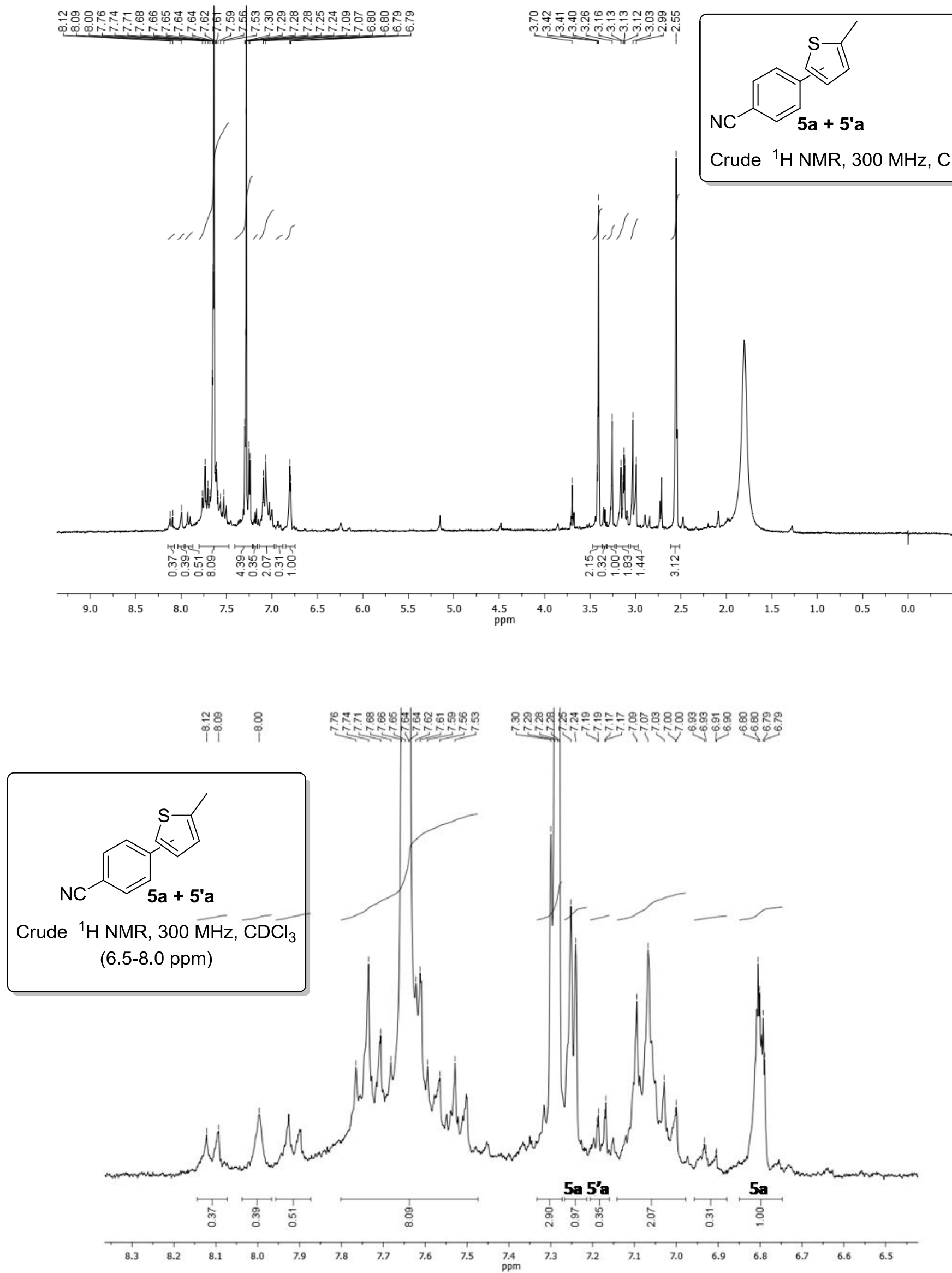

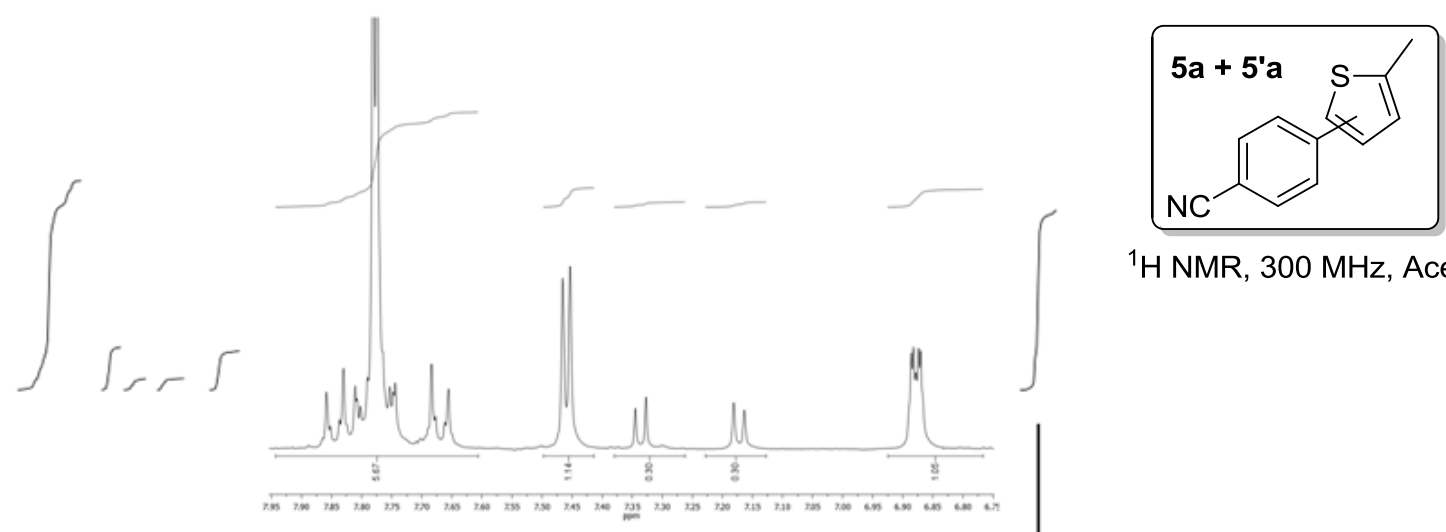

${ }^{1} \mathrm{H}$ NMR, $300 \mathrm{MHz}$, Acetone- $\mathrm{d}_{6}$

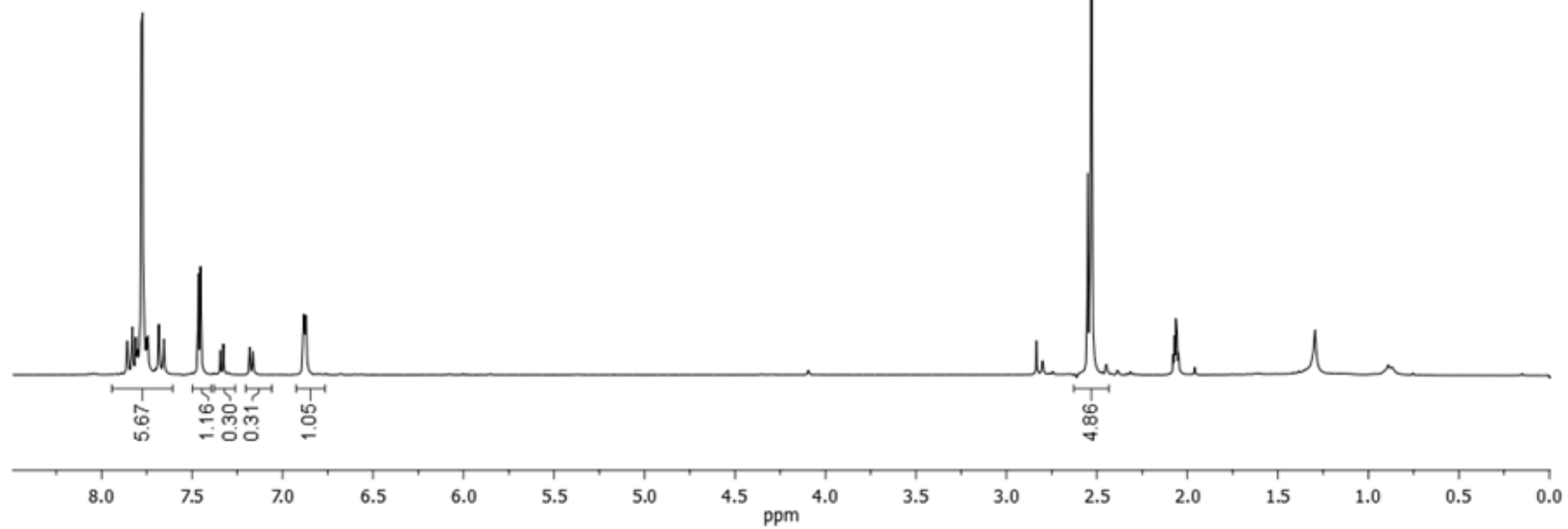

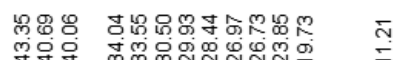

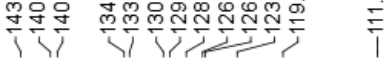

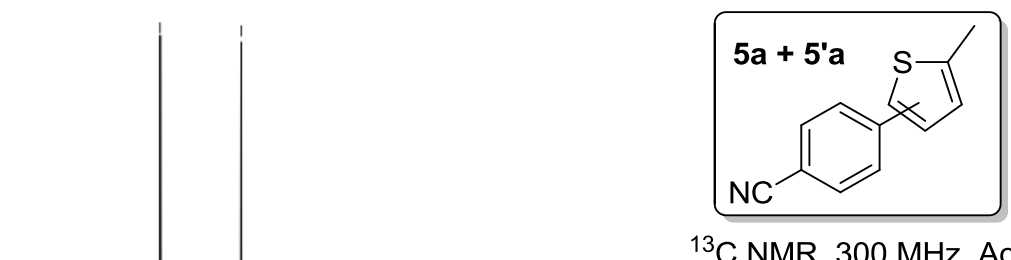

${ }^{13} \mathrm{C}$ NMR, $300 \mathrm{MHz}$, Acetone- $\mathrm{d}_{6}$

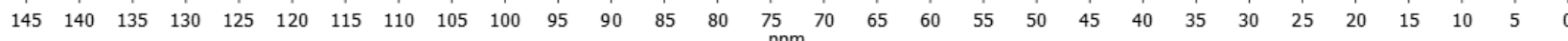




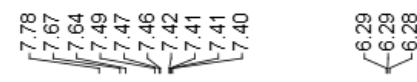<smiles>CCOC(=O)n1cccc1-c1ccc(C#N)cc1</smiles>

6a, ${ }^{1} \mathrm{H}$ NMR, $300 \mathrm{MHz}, \mathrm{CDCl}_{3}$

$119 \quad 5$

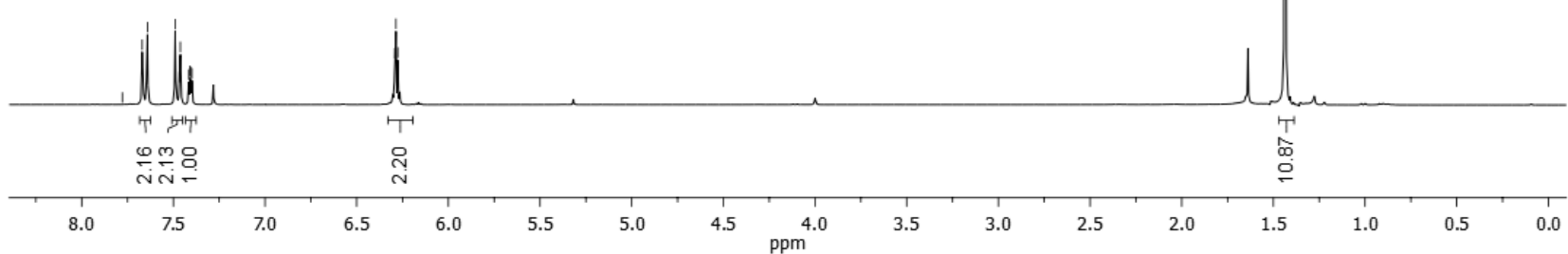

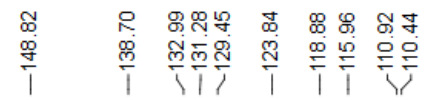

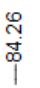
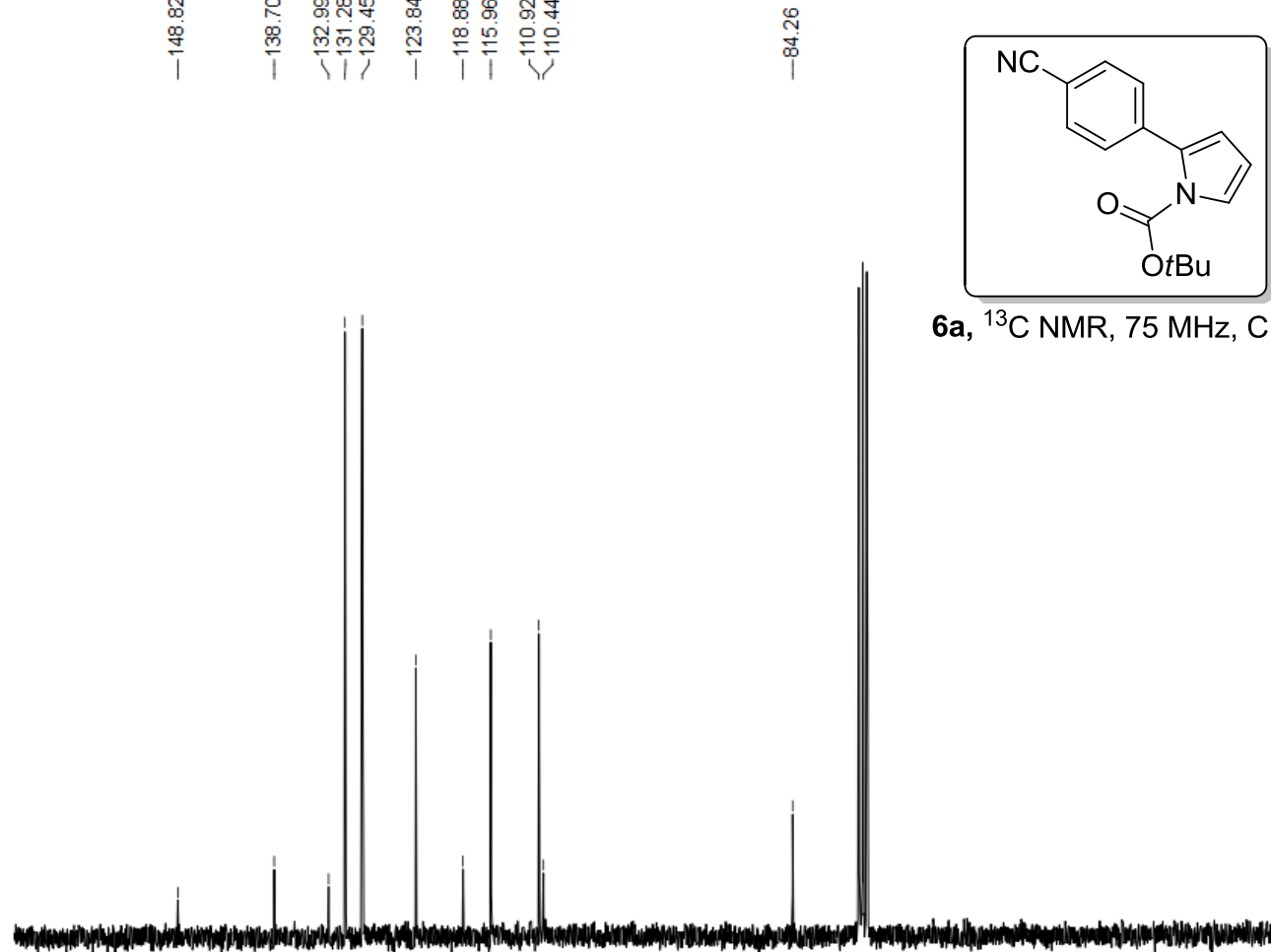

6a, ${ }^{13} \mathrm{C} \mathrm{NMR}, 75 \mathrm{MHz}, \mathrm{CDCl}_{3}$

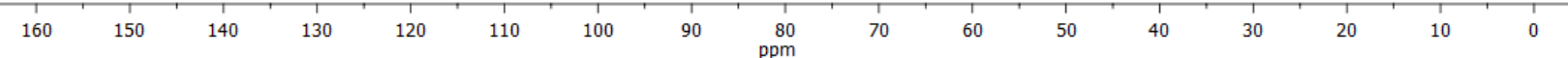



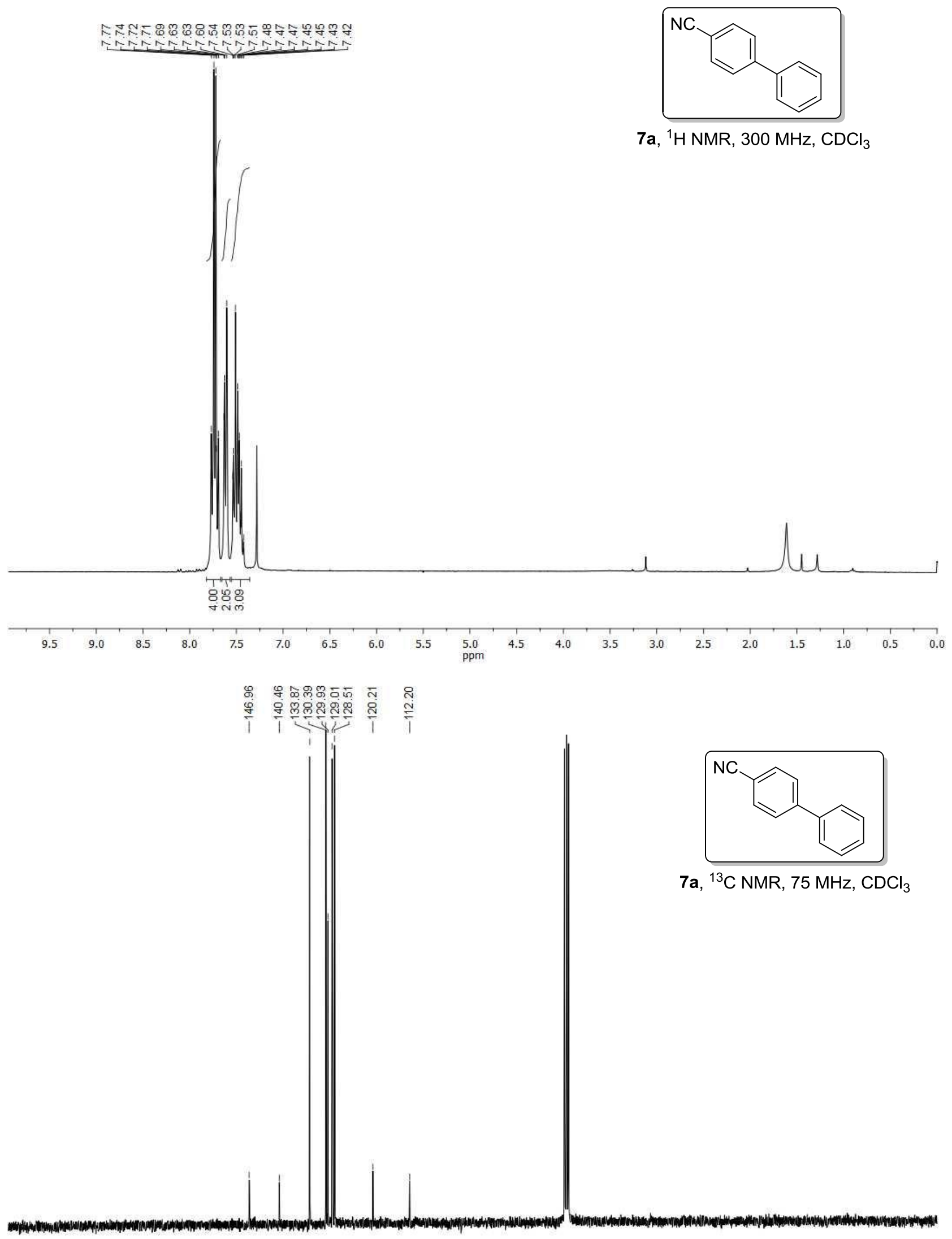

\begin{tabular}{|c|c|c|c|c|c|c|c|c|c|c|c|c|c|c|c|c|c|c|c|}
\hline 190 & 180 & 170 & 160 & 150 & 140 & 130 & 120 & 110 & $\begin{array}{c}100 \\
\mathrm{ppm}\end{array}$ & 90 & 80 & 70 & 60 & 50 & 40 & 30 & 20 & 10 & 0 \\
\hline
\end{tabular}




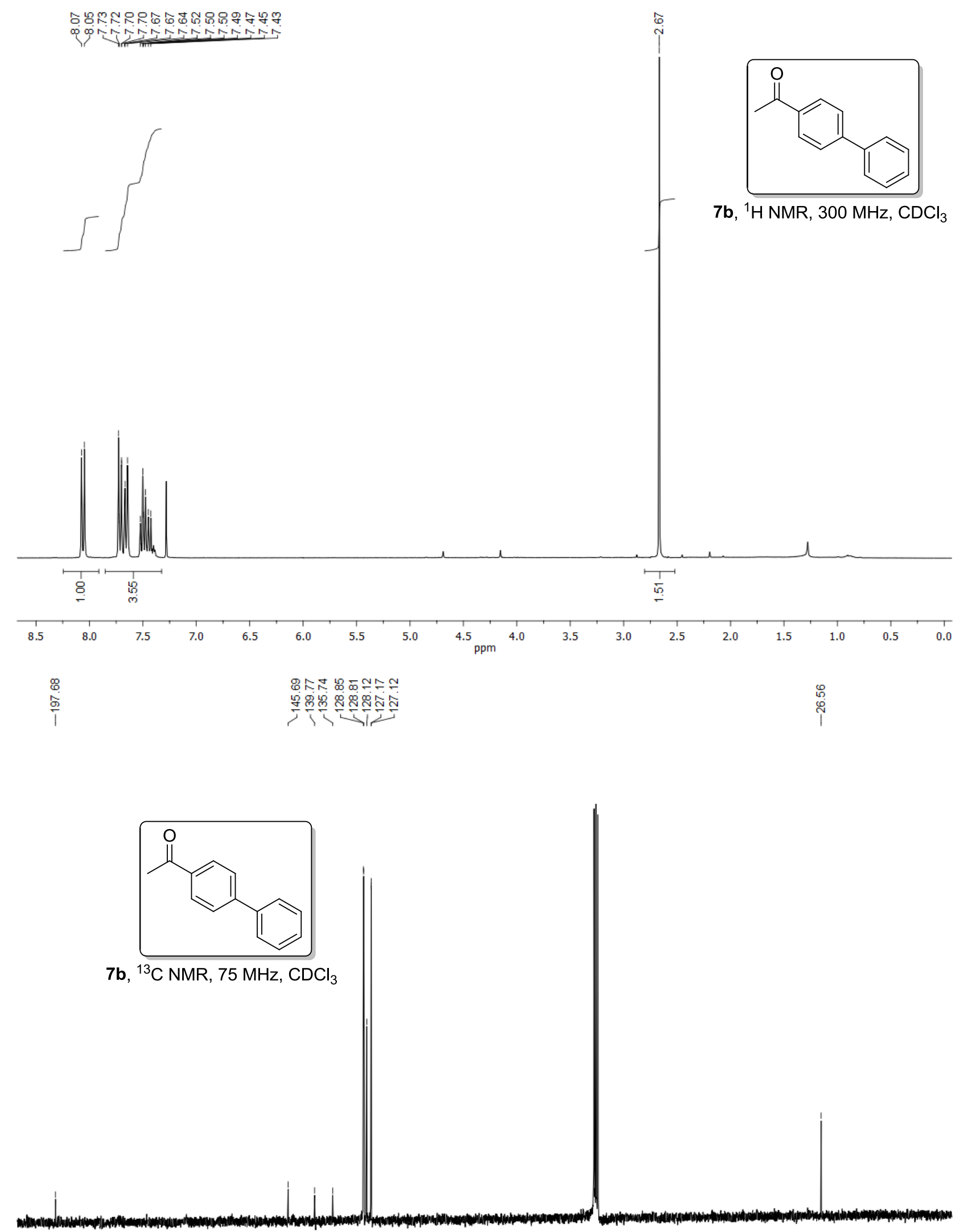

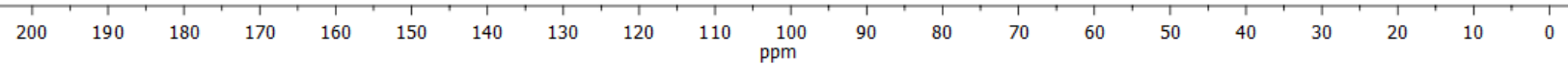




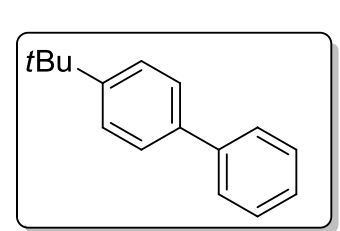

7e, ${ }^{1} \mathrm{H} \mathrm{NMR}, 300 \mathrm{MHz}, \mathrm{CDCl}_{3}$

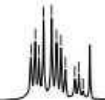

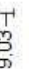

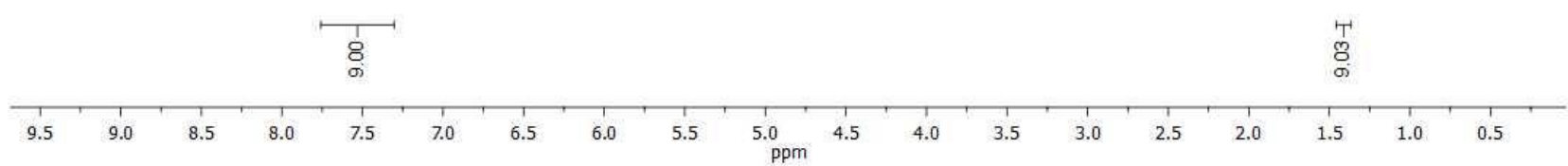

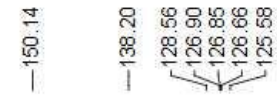

范

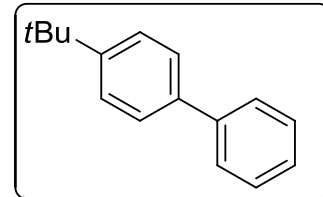

7e, ${ }^{13} \mathrm{C} \mathrm{NMR}, 75 \mathrm{MHz}, \mathrm{CDCl}_{3}$

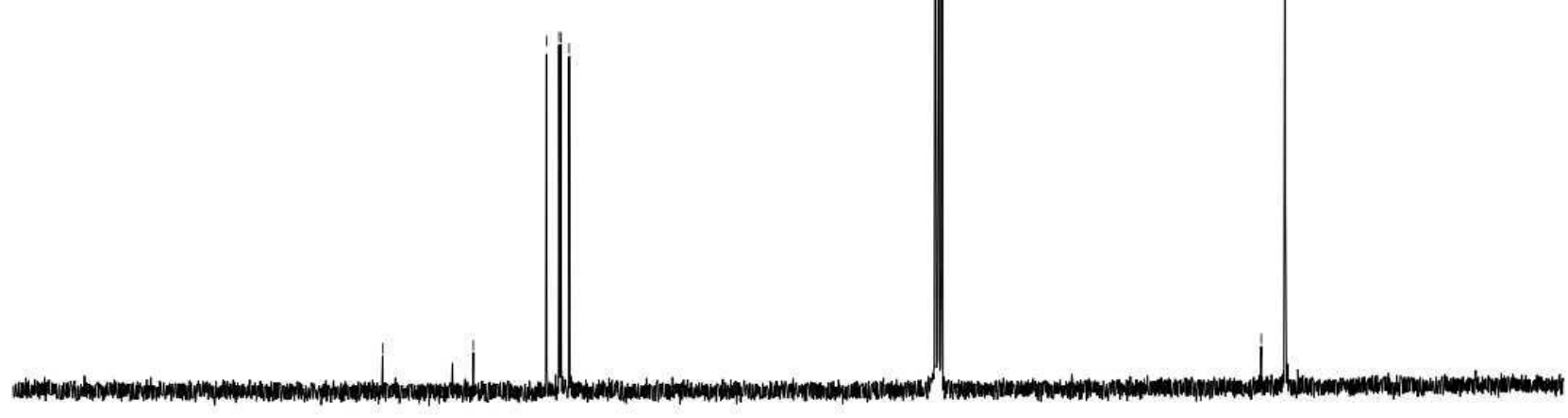

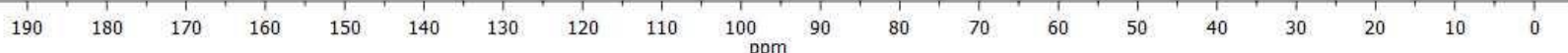



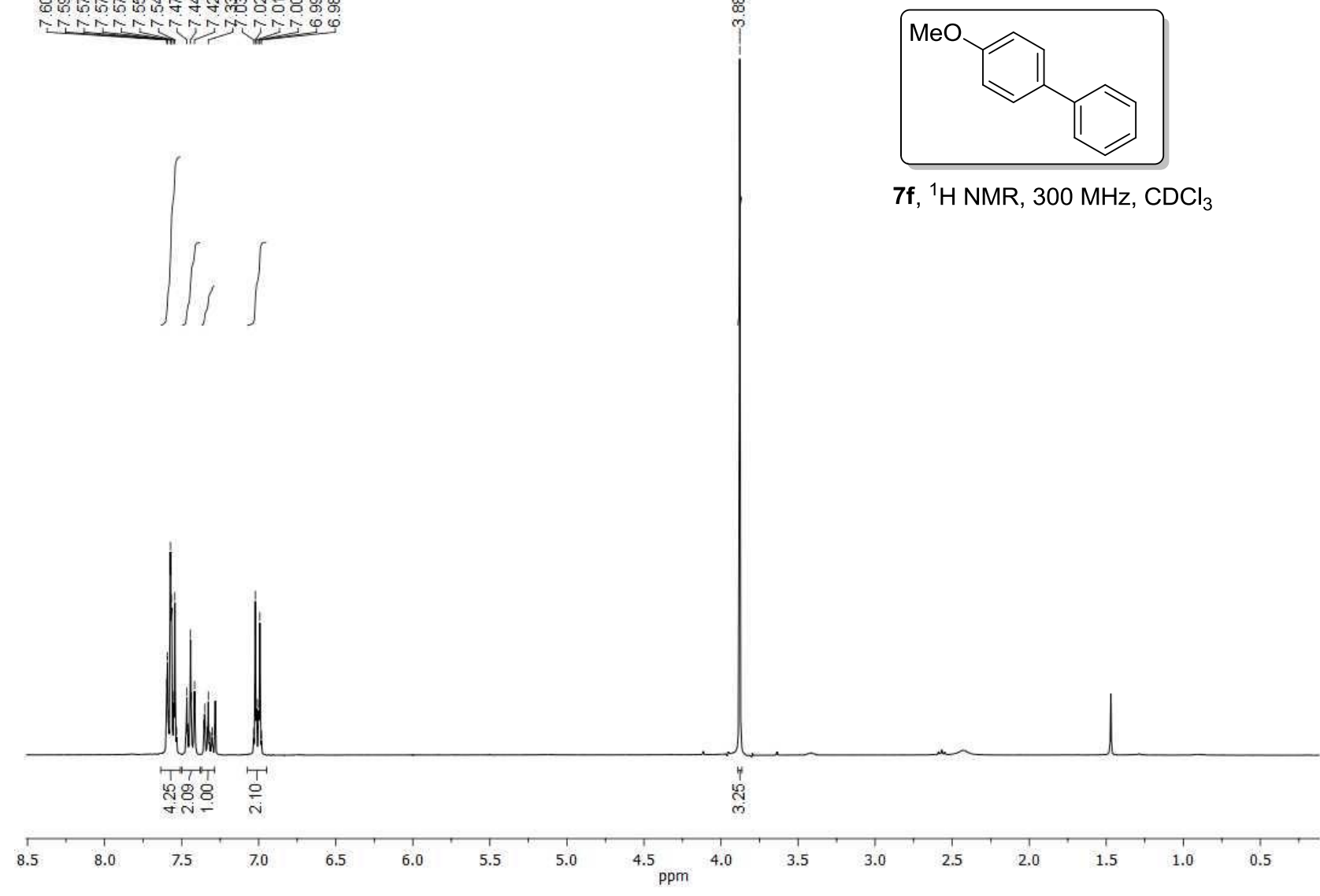

7f, ${ }^{1} \mathrm{H}$ NMR, $300 \mathrm{MHz}, \mathrm{CDCl}_{3}$

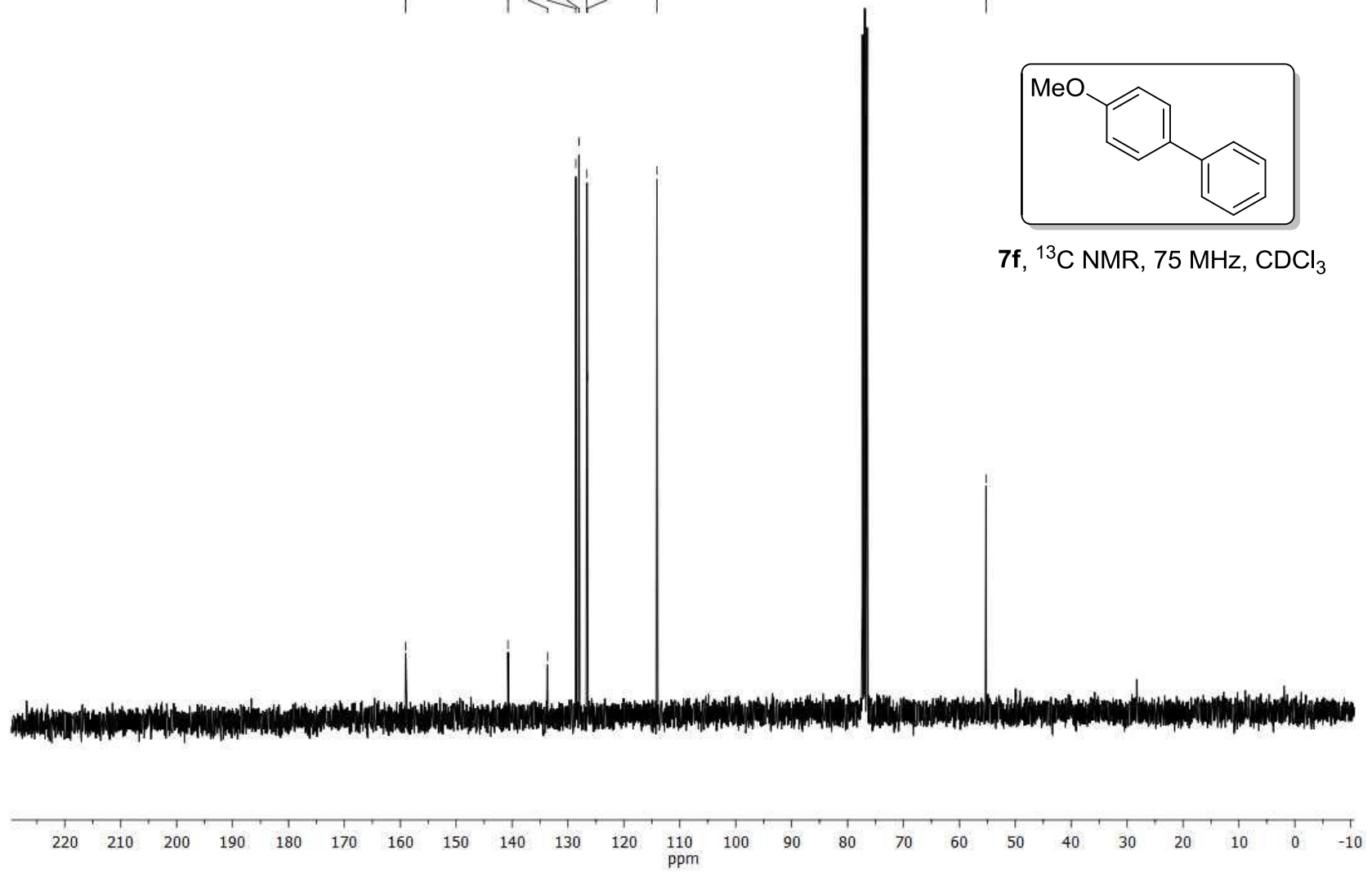




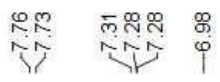
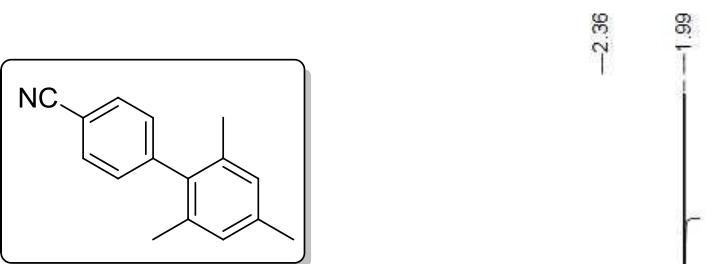

8a, ${ }^{1} \mathrm{H}$ NMR, $300 \mathrm{MHz}, \mathrm{CDCl}_{3}$

$\iiint$
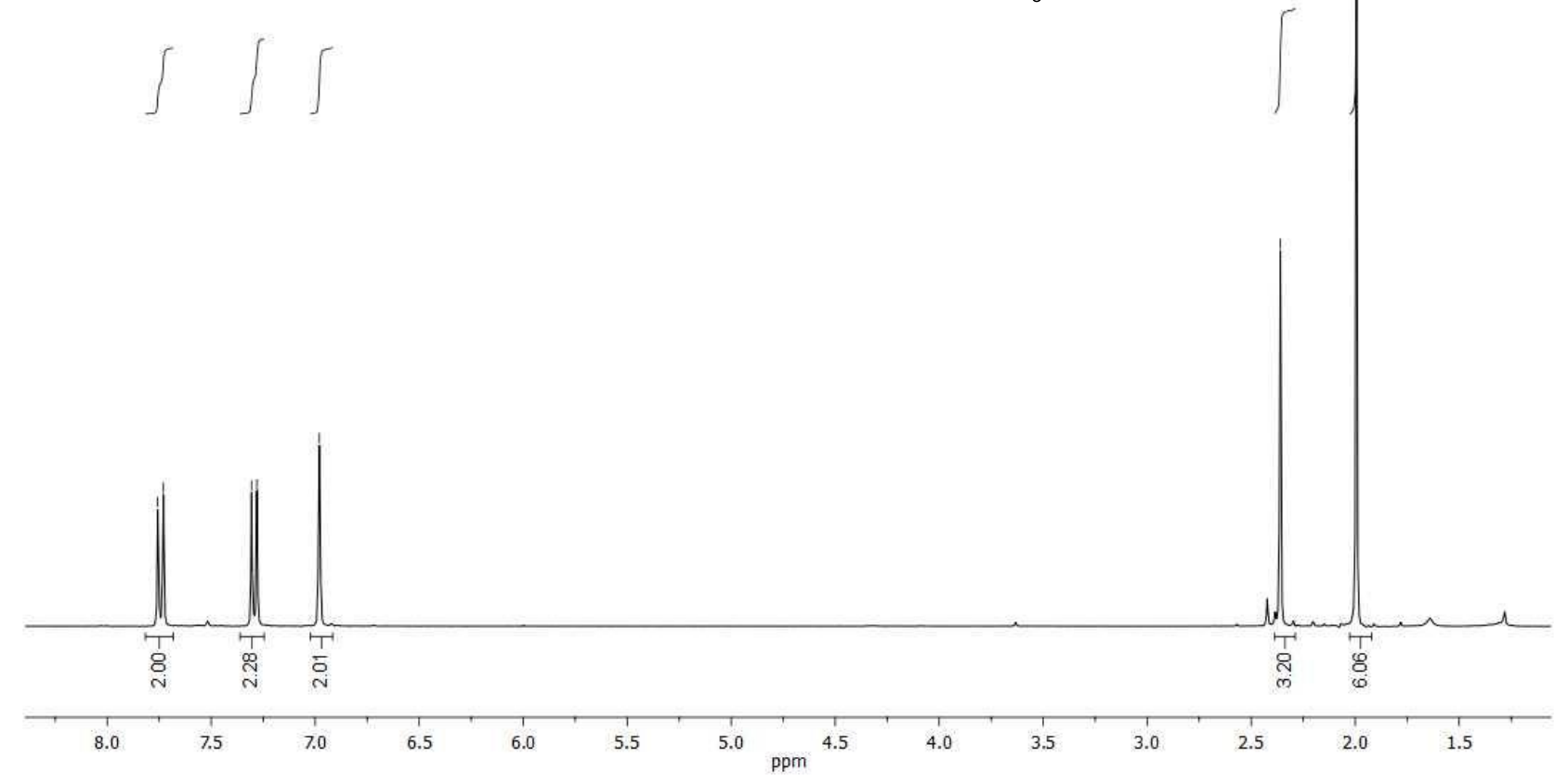

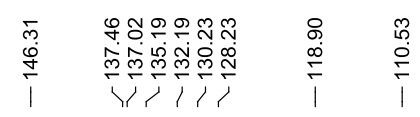
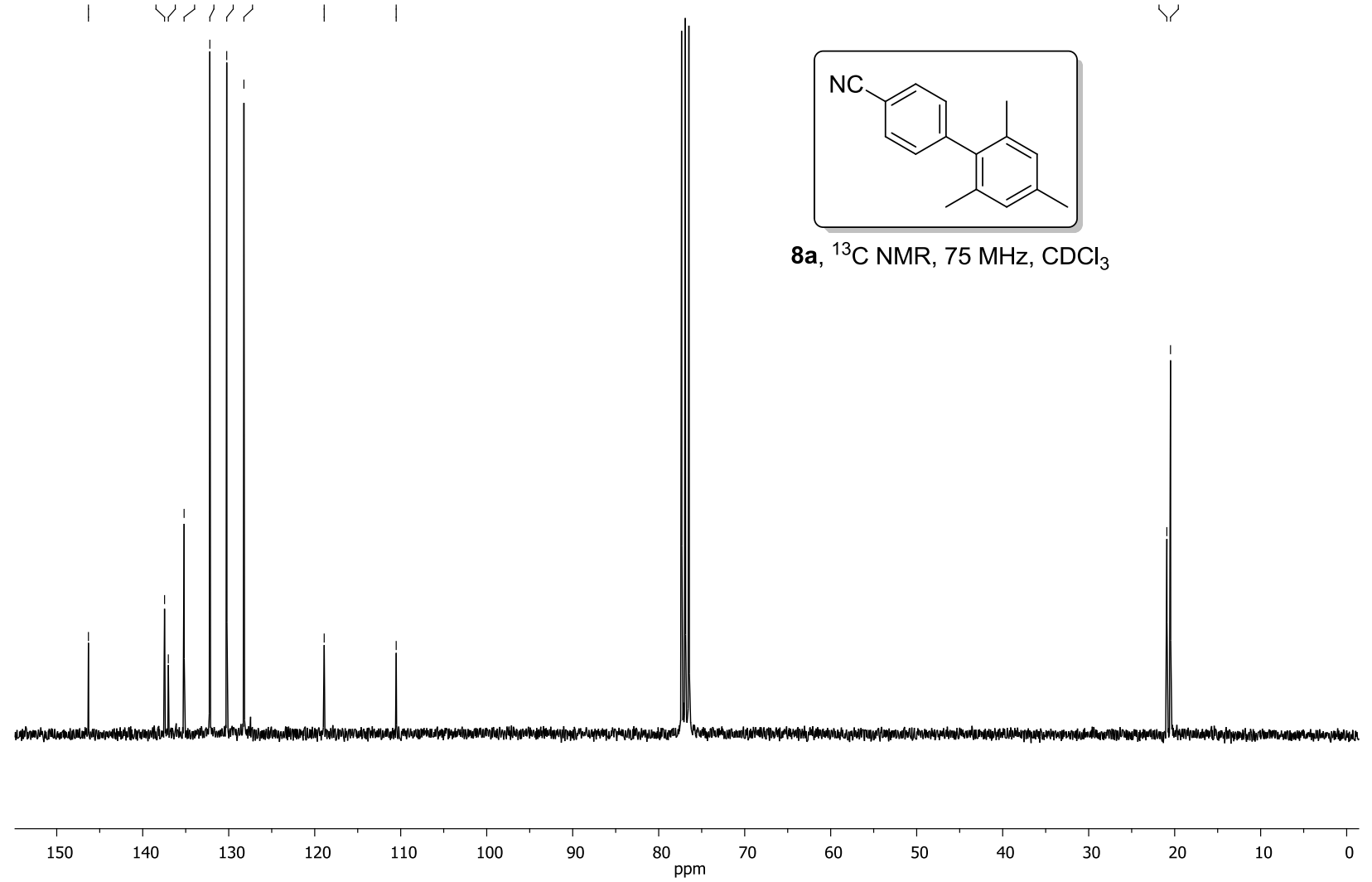

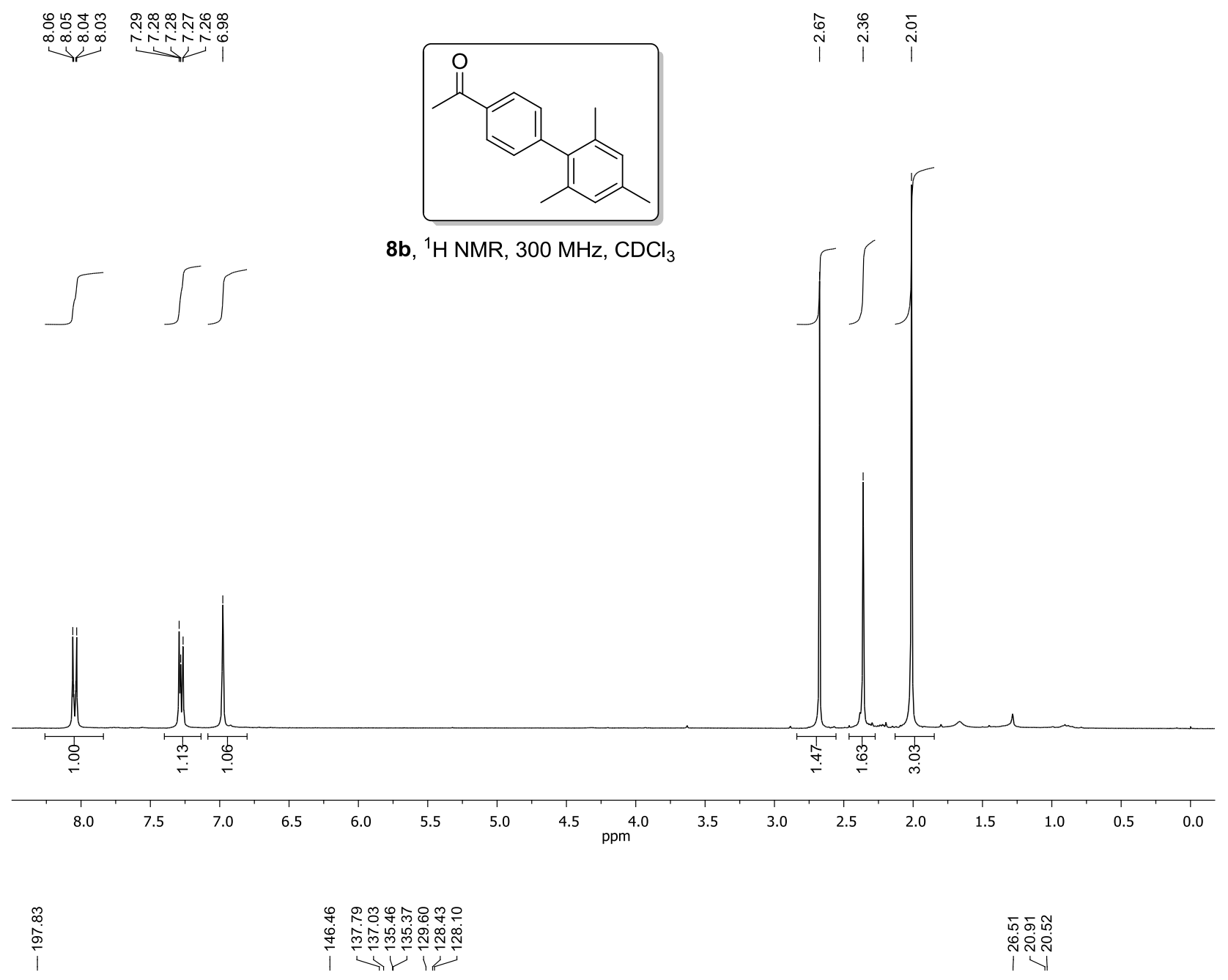

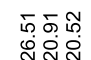

1

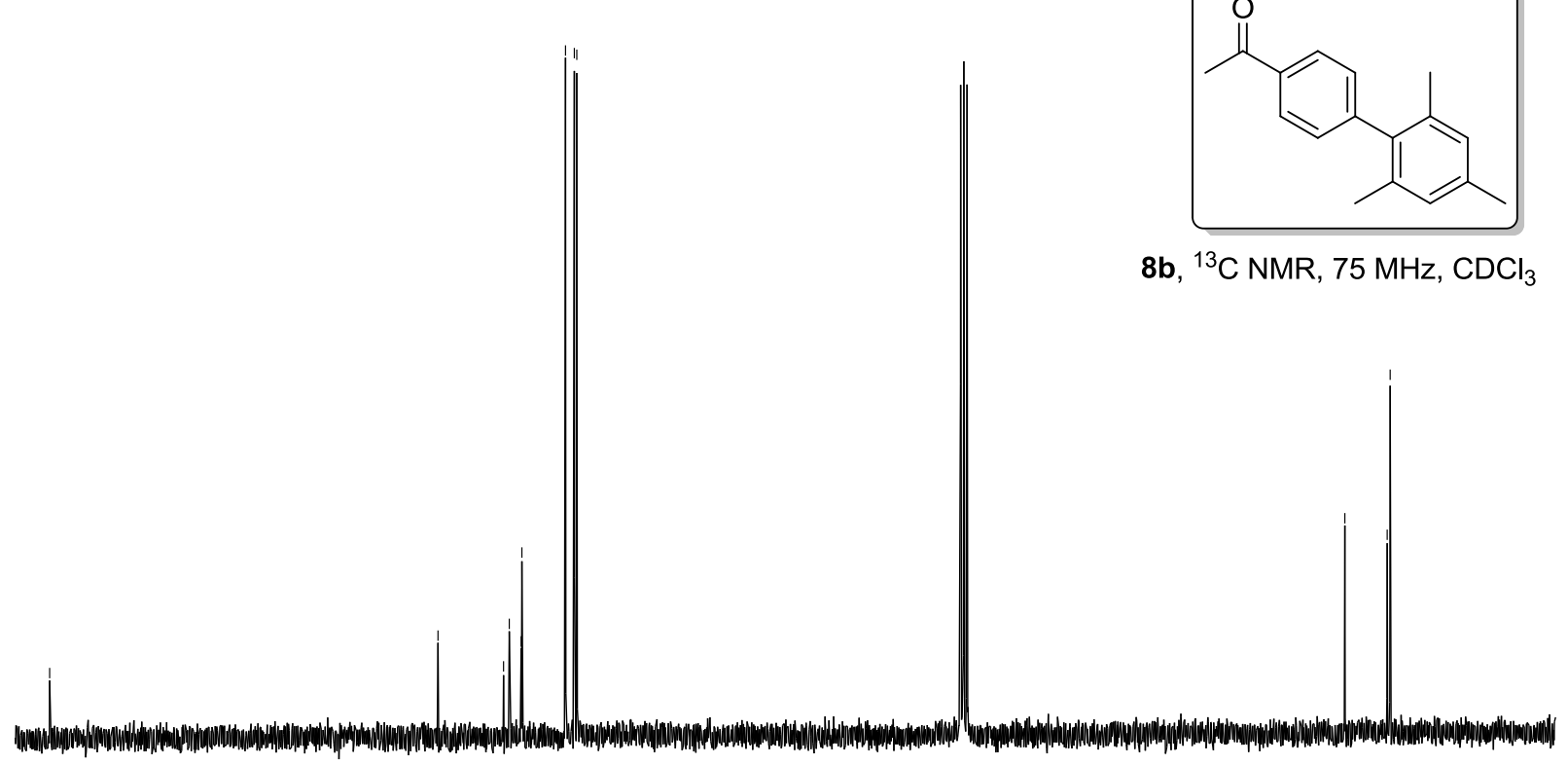

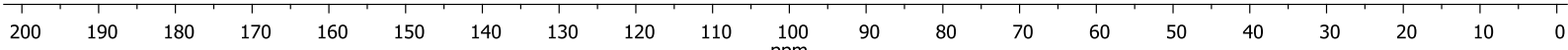

